\title{
The Ehrenfest approximation for electrons coupled to a phonon system
}

\author{
J le Page, D R Mason and W M C Foulkes \\ Department of Physics, Imperial College London, London, SW7 2AZ, UK \\ E-mail: jonathan.le-page@imperial.ac.uk
}

\begin{abstract}
We investigate the accuracy of the Ehrenfest approximation for a solid modelled as an independent electron system coupled to a many-mode phonon system. We construct a quantum to classical correspondence which we use to set the initial conditions of the Ehrenfest approximation given an initial density matrix. We find that the Ehrenfest approximation is accurate when the temperature of the phonon system is much higher than the temperature of the electron system. It is therefore reasonable to use the Ehrenfest approximation in the simulation of the later stages of radiation damage processes. We trace the failure of the Ehrenfest approximation to the absence of a term accounting for spontaneous phonon emission. The analytical work is validated numerically for the model system of a single oscillator coupled to electrons on a one-dimensional chain.
\end{abstract}

Submitted to: J. Phys.: Condens. Matter

\section{Introduction}

The Ehrenfest approximation is a simple approach to include non-adiabatic interactions between electrons and ions in time-dependent quantum mechanical simulations [1]. The ions are modelled as classical particles moving in a "mean-field" generated by the electrons. In turn the electrons are modelled as fully quantum mechanical particles evolving in a time-dependent potential generated by the ions. The Ehrenfest approximation has proved to be insufficient for modelling the scattering processes it was originally formulated for and has since been superseded by more complex methods $[2,3,4]$. Recently, though, materials modellers have been trying to include the non-adiabatic effects of electrons in simulations of solids, for example in attempts to model radiation damage [5]. A simple method is required if large scale simulations are to remain computationally tractable. In this paper we begin to investigate whether the Ehrenfest approximation is applicable to such simulations. For simplicity we shall assume that the motion of the ionic lattice can be modelled as a system of phonons.

Under the physically reasonable assumptions specified in section 2 we shall compare the energy transfer between the electrons and ions in full quantum mechanical simulations against that given by the corresponding Ehrenfest approximation. We find that the Ehrenfest approximation correctly accounts for the energy transfer when the ions have a much higher temperature than the electrons. The discrepancy between 
the approximated energy transfer and that given by full quantum mechanics is traced to the failure of the Ehrenfest approximation to include the spontaneous emission of phonons.

The outline of the paper is as follows. In section 2 we state the Hamiltonian we use to model a solid and specify the initial conditions. In section 3 we introduce a quantum to classical correspondence which will allow us to relate the initial quantum mechanical state of the ions to a set of initial conditions of the classical ions in the Ehrenfest simulation. In sections 4 and 5 we approach the electron evolution analytically to determine the cases in which the Ehrenfest approximation fails. This analytical work is based on the notion that the motion of the ions is not affected significantly by the electrons and so each mode acts as a time-dependent oscillating potential on the electrons. In section 6 the assumptions and derivations made in this paper are validated numerically for a simple model system. Finally, in section 7 , we state our conclusions.

\section{System Hamiltonian}

We choose to model a solid as a coupled many electron, many oscillator system. The resulting Hamiltonian, derived and discussed in a number of texts $[6,7,8]$, is given by

$$
\begin{aligned}
\hat{H}= & \hat{H}_{e}+\hat{H}_{I}+\hat{H}_{e I} \\
= & \sum_{\mathbf{k}, \sigma} \epsilon_{\sigma}(\mathbf{k}) \hat{c}_{\sigma}^{+}(\mathbf{k}) \hat{c}_{\sigma}(\mathbf{k})+\sum_{\mathbf{q}, \lambda} \hbar \omega_{\lambda}(\mathbf{q})\left[\hat{a}_{\lambda}^{+}(\mathbf{q}) \hat{a}_{\lambda}(\mathbf{q})+\frac{1}{2}\right] \\
& +\sum_{\mathbf{k}, \mathbf{k}^{\prime}, \lambda} g_{\lambda}\left(\mathbf{k}, \mathbf{k}^{\prime}\right) \hat{c}_{\sigma}^{+}\left(\mathbf{k}^{\prime}\right) \hat{c}_{\sigma}(\mathbf{k})\left[\hat{a}_{\lambda}(\mathbf{q})+\hat{a}_{\lambda}^{+}(-\mathbf{q})\right] .
\end{aligned}
$$

Here $\hat{c}_{\sigma}^{+}(\mathbf{k})$ is the creation operator for an electron with Bloch momentum $\mathbf{k}$ in spin $\sigma$; $\epsilon_{\sigma}(\mathbf{k})$ denotes the energy of this state; $\hat{a}_{\lambda}^{+}(\mathbf{q})$ is the creation operator for a phonon with wavevector $\mathbf{q}$ with polarisation $\lambda$ and $\omega_{\lambda}(\mathbf{q})$ is the corresponding angular frequency. We have used $\mathbf{q}=\mathbf{k}^{\prime}-\mathbf{k}+\tilde{\mathbf{k}}$ where $\tilde{\mathbf{k}}$ is a vector of the reciprocal lattice such that $\mathbf{q}$ lies in the first Brillouin zone. The amplitude of this mode is defined as

$$
\hat{Q}_{\lambda}(\mathbf{q})=\left(\frac{\hbar}{2 m \omega_{\lambda}(\mathbf{q})}\right)^{\frac{1}{2}}\left[\hat{a}_{\lambda}(\mathbf{q})+\hat{a}_{\lambda}^{+}(-\mathbf{q})\right],
$$

where for simplicity we have assumed that all ions involved have the same mass $m$. The electron-phonon coupling matrix elements are denoted $g_{\lambda}\left(\mathbf{k}, \mathbf{k}^{\prime}\right)$. They contain all the information regarding the interaction between the electrons and phonons. Throughout this paper we assume only a single band of electrons and neglect spin to simplify notation.

We mention a number of important assumptions made in the derivation of (1) to highlight the limits of its validity:

(i) The electrons of the system are non-interacting.

(ii) The displacements of the ions from their equilibrium sites are small. We can thus describe the ionic system as independent oscillating modes. Furthermore, if the rigid ion approximation holds, we can assume that the electron-ion coupling is linear in displacement.

(iii) The equilibrium positions of the ions form a lattice with translation symmetry. This allows us to use Bloch's theorem to label the electron eigenstates and the oscillator modes. 
For the analysis made in this paper we will also require the additional assumption that the coupling term $\hat{H}_{\mathrm{eI}}$ is small. We can then use linear response theory.

We denote the eigenstates of the uncoupled system by

$$
|\mathbf{K} \boldsymbol{\Phi}\rangle=\left|n_{\mathbf{k}_{1}} \ldots n_{\mathbf{k}_{D}} N_{\mathbf{q}_{1}, \lambda_{1}} N_{\mathbf{q}_{2}, \lambda_{2}} \ldots N_{\mathbf{q}_{3 \mathcal{N}}, \lambda_{3 \mathcal{N}}}\right\rangle,
$$

where $\mathbf{K}$ denotes an eigenstate of the uncoupled many-electron system, $\mathbf{\Phi}$ is an eigenstate of the uncoupled many-ion system, $D$ is the number of eigenstates of the electron system and $\mathcal{N}$ is the number of ions. Since both systems are noninteracting we write these eigenstates in the occupation number representation: $n_{\mathbf{k}}$ is the occupation number for single electron eigenstate $\mathbf{k}$ and $N_{\mathbf{q}, \lambda}$ is the phonon number of the mode with wavevector $\mathbf{q}$ and polarisation $\lambda$. The eigenstates have corresponding energies $E_{\mathbf{K}}$ and $W_{\boldsymbol{\Phi}}$ where

$$
E_{\mathbf{K}}=\sum_{\substack{\mathbf{k} \\ \text { occ }}} \varepsilon(\mathbf{k}), W_{\mathbf{\Phi}}=\sum_{\mathbf{q}, \lambda} \hbar \omega_{\lambda}(\mathbf{q})\left(N_{\mathbf{q}, \lambda}+\frac{1}{2}\right) .
$$

Furthermore we define $E_{\mathbf{K} \boldsymbol{\Phi}}=E_{\mathbf{K}}+W_{\boldsymbol{\Phi}}$.

We specify that the system starts in an initial state where the electrons and ions have been set to separate temperatures $T_{\mathrm{e}}$ and $T_{\mathrm{ion}}$,

$$
\hat{\rho}=\sum_{\mathbf{K}} \frac{1}{Z_{\mathrm{e}}} \exp \left(-\frac{E_{\mathbf{K}}}{k_{\mathrm{B}} T_{\mathrm{e}}}\right) \sum_{\boldsymbol{\Phi}} \frac{1}{Z_{\text {ion }}} \exp \left(-\frac{W_{\boldsymbol{\Phi}}}{k_{\mathrm{B}} T_{\text {ion }}}\right)|\mathbf{K} \mathbf{\Phi}\rangle\langle\mathbf{K} \mathbf{\Phi}|,
$$

where $Z_{\mathrm{e}}$ and $Z_{\text {ion }}$ are the partition functions for the electron and ion systems, respectively, and $k_{\mathrm{B}}$ is Boltzmann's constant. The use of temperature here is not meant to suggest the physical situation of two systems in equilibrium being brought together. Rather we use 'temperature' as a means to parameterise the electronic and ionic energy scales for our analytic calculations.

\section{A Quantum to Classical Correspondence}

In a system modelled by the Ehrenfest approximation the electron system is represented by a quantum mechanical density matrix, $\hat{\rho}_{\mathrm{e}}(t)$, and the ion system is given by a point $(\mathbf{R}(t), \mathbf{P}(t))$ in the $6 \mathcal{N}$ dimensional phase space. We use a subscript $i$ to denote the position and momentum of the $i$ th ion and $\hat{\imath}$ to indicate quantum mechanical operators. To derive the Ehrenfest approximation we note that $\hat{H}_{\mathrm{I}}$ and $\hat{H}_{\mathrm{eI}}$ may be written in terms of the ionic position and momentum operators as

$$
\begin{aligned}
\hat{H}_{\mathrm{I}} & =\sum_{i} \frac{\hat{\mathbf{P}}_{i}^{2}}{2 m}+\sum_{i, j} \frac{\partial^{2} V_{\mathrm{II}}}{\partial \mathbf{R}_{i} \partial \mathbf{R}_{j}}\left(\mathbf{R}_{i}^{0}, \mathbf{R}_{j}^{0}\right)\left(\hat{\mathbf{R}}_{i}-\mathbf{R}_{i}^{0}\right) \cdot\left(\hat{\mathbf{R}}_{j}-\mathbf{R}_{j}^{0}\right) \\
& =\sum_{i, \alpha} \frac{\hat{\mathbf{P}}_{i}^{2}}{2 m}+V_{\mathrm{I}}(\hat{\mathbf{R}}) \\
\hat{H}_{\mathrm{eI}} & =\sum_{i \alpha} \sum_{\mathbf{k}, \mathbf{k}^{\prime}} \hat{c}^{+}\left(\mathbf{k}^{\prime}\right) \hat{c}(\mathbf{k})\left\langle\mathbf{k}^{\prime}, \nabla_{\mathbf{R}_{i}} V_{\mathrm{eI}}\left(\hat{\mathbf{r}}_{\mathrm{e}}-\mathbf{R}_{i}^{0}\right) \mathbf{k}\right\rangle \cdot\left(\hat{\mathbf{R}}_{i}-\mathbf{R}_{i}^{0}\right)
\end{aligned}
$$

where $\mathbf{R}_{i}^{0}$ denotes the ion's equilibrium position. $\hat{V}_{\text {II }}$ denotes the ion-ion interaction and $\hat{V}_{\mathrm{eI}}$ the electron-ion interaction. $\hat{\mathbf{r}}_{\mathrm{e}}$ denotes the electronic position operator. (See [7] for details.) Note that the operator $\hat{V}_{\mathrm{I}}$ is defined by the above relation. The 
Ehrenfest approximation involves replacing the ionic position operator with classical trajectories and evolving the system by the following $\mathcal{N}+1$ coupled equations

$$
\begin{aligned}
& \mathrm{i} \hbar \dot{\hat{\rho}}_{\mathrm{e}}(t)=\left[\hat{H}_{\mathrm{e}}+\hat{H}_{\mathrm{eI}}(\mathbf{R}(t)), \hat{\rho}_{\mathrm{e}}(t)\right], \\
& m \ddot{\mathbf{R}}_{i}(t)=-\nabla_{\mathbf{R}_{i}} V(\mathbf{R}(t))-\operatorname{Tr}\left\{\hat{\rho}_{\mathrm{e}}(t)\left(\nabla_{\mathbf{R}_{i}} \hat{H}_{\mathrm{eI}}(\mathbf{R}(t))\right)\right\} .
\end{aligned}
$$

Here $\hat{H}_{\mathrm{e}}$ is the electronic Hamiltonian acting only on the electronic wavefunction, $-\nabla_{\mathbf{R}_{i}} V(\mathbf{R}(t))$ is the inter-ion force (a real valued function on the ionic phase space) and $\hat{H}_{\mathrm{eI}}(\mathbf{R}(t))$ is an operator parameterised by the ionic position which couples the two systems. The initial density matrix, denoted $\hat{\rho}_{\mathrm{e}}$, is the density matrix of the electronic system only. (The form of (3) is such that the density matrix separates in to independent electron and phonon parts.)

In order to make an Ehrenfest approximation we must deal with the additional "mathematical structure" of quantum mechanics over classical mechanics. This additional structure means that there is no specific one-to-one relation between a point in phase space and a wavefunction. For example, there is only one point in phase space with position and momentum zero but all the eigenfunctions of a quantum simple harmonic oscillator have position and momentum expectation values of zero. To relate Ehrenfest simulations to full quantum mechanics it is necessary to determine the most sensible and useful mapping between some subset of quantum mechanical states and the classical phase space used in the Ehrenfest approximation. In this paper we use the following correspondence:

The presence of a mode q with polarisation $\lambda$ containing an average of $\left\langle N_{\mathbf{q}, \lambda}\right\rangle$ phonons is modelled by an Ehrenfest simulation where the corresponding classical mode has an initial amplitude such that

$$
\frac{1}{2} m \omega_{\lambda}^{2}(\mathbf{q}) A_{\mathbf{q}, \lambda}^{2}=\hbar \omega_{\lambda}(\mathbf{q})\left(\frac{\sqrt{\left\langle N_{\mathbf{q}, \lambda}\right\rangle}+\sqrt{\left\langle N_{-\mathbf{q}, \lambda}\right\rangle}}{2}\right)^{2} .
$$

The rationale for this scheme comes from the theory of coherent states $[9,10]$. Coherent states are quantum states for which the Heisenberg uncertainty relation between the position and momentum observables has it lowest possible value. Thus, a coherent state is, in some sense, as "classical" as a quantum mechanical state can be.

For exposition purposes we first consider the coherent states of a single oscillator. Let $\hat{a}^{+}$and $\hat{a}$ be the creation and annihilation operators of a phonon. For a given complex number, $z$, we define the coherent state

$$
|z\rangle:=\exp \left(-\frac{|z|^{2}}{2}\right) \exp \left(z \hat{a}^{+}\right)|0\rangle
$$

where $|0\rangle$ is the vacuum state. For this work a coherent state has three important properties.

(i) The expectation value of the total number of phonons in the system is given by $|z|^{2}$. Additionally the mean square fluctuation of the phonon number operator decreases in relative terms as the average number of phonons increases,

$$
\frac{\left\langle z,\left(\hat{a}^{+} \hat{a}-\left\langle\hat{a}^{+} \hat{a}\right\rangle\right)^{2} z\right\rangle}{\left(\left\langle\hat{a}^{+} \hat{a}\right\rangle\right)^{2}}=\frac{1}{|z|^{2}}=\frac{1}{\left\langle\hat{a}^{+} \hat{a}\right\rangle} .
$$


(ii) The expectation values of the position and momentum observables of the simple harmonic oscillator, $\hat{x}$ and $\hat{p}$, are proportional to the real and imaginary parts of $z$,

$$
\begin{aligned}
& \langle z, \hat{x} z\rangle=\sqrt{\frac{\hbar}{2 m \omega}}\left\langle z,\left(\hat{a}^{+}+\hat{a}\right) z\right\rangle=\sqrt{\frac{2 \hbar}{m \omega}} \Re\{z\}, \\
& \langle z, \hat{p} z\rangle=\mathrm{i} \sqrt{\frac{m \hbar \omega}{2}}\left\langle z,\left(\hat{a}^{+}-\hat{a}\right) z\right\rangle=\sqrt{2 m \hbar \omega} \Im\{z\} .
\end{aligned}
$$

(iii) Under the evolution of the simple harmonic oscillator Hamiltonian the position and momentum expectation values for any state obey the classical equation of motion

$$
\langle\ddot{\hat{x}}\rangle(t)+\omega^{2}\langle\hat{x}\rangle(t)=0 .
$$

If we assume that the initial state of the system is the coherent state $|z\rangle$, where $z=A$ is a real number, then the time-dependence of the expectation value of position is given by

$$
\langle\hat{x}\rangle(t)=A \cos (\omega t) .
$$

Note that choosing $z$ to be a real number simply sets the initial phase; any complex number with the same magnitude will evolve with the same amplitude and angular frequency.

If the oscillator is initially in an eigenstate of $\hat{H}_{\mathrm{I}}$ with a large number of phonons and the observable we are considering depends only on the phonon number, property (i) allows us to consider a coherent state with $z$ such that $|z|^{2}=N$ instead. Property (iii) then indicates that we can model the expected position and momenta of this state classically. Furthermore, by (ii), we find the amplitude of the oscillation, $A$, is related to the phonon number in the following way

$$
A=\left(\frac{2 \hbar}{m \omega}\right)^{\frac{1}{2}}|z|=\left(\frac{2 \hbar}{m \omega} N\right)^{\frac{1}{2}} \Rightarrow \frac{1}{2} m \omega^{2} A^{2}=\hbar \omega N
$$

Thus, in this simple one dimensional case, we see that by establishing an equivalence between eigenstates and coherent states with the same expected number of phonons, we specify a quantum to classical correspondence.

The above argument can be extended to the multiple mode case by considering a coherent state of the form

$$
\left|z_{\mathbf{q}_{1}, \lambda_{1}}, z_{\mathbf{q}_{2}, \lambda_{2}}, \ldots, z_{\mathbf{q}_{3 \mathcal{N}}, \lambda_{3 \mathcal{N}}}\right\rangle:=\exp \left(-\frac{1}{2} \sum_{\mathbf{q}, \lambda}\left|z_{\mathbf{q}, \lambda}\right|^{2}\right) \exp \left(\sum_{\mathbf{q}, \lambda} z_{\mathbf{q}, \lambda} \hat{a}_{\lambda}^{+}(\mathbf{q})\right)|0\rangle
$$

for the $3 \mathcal{N}$ complex numbers $z_{\mathbf{q}_{1}, \lambda_{1}}, \ldots, z_{\mathbf{q}_{3 \mathcal{N}}, \lambda_{3 \mathcal{N}}}$. We can then use $(2)$ and a method identical to the one-dimensional case to determine the amplitudes of the modes of an initial classical state equivalent to the quantum state $\left|N_{\mathbf{q}_{1}, \lambda_{1}} N_{\mathbf{q}_{2}, \lambda_{2}} \ldots N_{\mathbf{q}_{3 \mathcal{N}}, \lambda_{3 \mathcal{N}}}\right\rangle$ leading to (6). Having established the initial amplitude of each mode the periodicity of the crystal structure allows us to determine the initial position and momenta through Fourier transforms. 


\section{Semiclassical Hamiltonian}

We wish to model a quantum mechanical system, with Hamiltonian of the form (1) with initial condition (3), via the Ehrenfest approximation. To do so we first apply the quantum to classical correspondence detailed in section 3 in order to set the initial state of the classical ions. We then evolve the system according to equations (4) and (5). As the electrons evolve they alter the potential through which the ions move, affecting their evolution via the "mean-field" term in (5). In this way the Ehrenfest approximation contains a feedback between the electrons and the ions which make the equations difficult to approach analytically.

For systems with weak electron-ion coupling the effect of the electrons on the classical ion behaviour is small and each ionic mode just oscillates with the frequency of that mode in the uncoupled system. We assume that the effect of the electrons on the ions is so small as to be insignificant so that even when the two systems are coupled each mode still just oscillates as in the uncoupled system. The time-dependence of the amplitude of each mode with initial condition (6) is then given by

$Q_{\lambda}(\mathbf{q})=\left(\frac{\hbar}{2 m \omega_{\lambda}(\mathbf{q})}\left\langle N_{\mathbf{q}, \lambda}\right\rangle\right)^{\frac{1}{2}} \mathrm{e}^{-\mathrm{i} \omega_{\lambda}(\mathbf{q}) t}+\left(\frac{\hbar}{2 m \omega_{\lambda}(\mathbf{q})}\left\langle N_{-\mathbf{q}, \lambda}\right\rangle\right)^{\frac{1}{2}} \mathrm{e}^{\mathrm{i} \omega_{\lambda}(-\mathbf{q}) t}$,

ensuring that the ion positions are real-valued. In the following analysis we therefore neglect the explicit evolution of the ions given by (5) and focus only on the evolution of the electrons. By replacing the amplitude operator of (1) with (8) we obtain the following time-dependent Hamiltonian

$$
\begin{aligned}
& \hat{H}^{\mathrm{sc}}(t)=\sum_{\mathbf{k}, \sigma} \epsilon_{\sigma}(\mathbf{k}) \hat{c}_{\sigma}^{+}(\mathbf{k}) \hat{c}_{\sigma}(\mathbf{k}) \\
& +\sum_{\mathbf{k}, \mathbf{k}^{\prime}, \lambda} g_{\lambda}\left(\mathbf{k}, \mathbf{k}^{\prime}\right) \hat{c}_{\sigma}^{+}\left(\mathbf{k}^{\prime}\right) \hat{c}_{\sigma}(\mathbf{k})\left[\sqrt{\left\langle N_{\mathbf{q}, \lambda}\right\rangle} \mathrm{e}^{-\mathrm{i} \omega_{\lambda}(\mathbf{q}) t}+\sqrt{\left\langle N_{-\mathbf{q}, \lambda}\right\rangle} \mathrm{e}^{\mathrm{i} \omega_{\lambda}(-\mathbf{q}) t}\right] .
\end{aligned}
$$

We call this the semiclassical Hamiltonian in analogy with the semiclasical approximation of photons in quantum optics. The assumptions that we have made in section 2 mean that the electronic evolution given by the semiclassical Hamiltonian will be almost the same as that given by the Ehrenfest approximation. Note that where, in the full quantum simulation the state of the phonon system is specified by the initial condition (3), in the semiclassical simulation this is reflected in the amplitudes of the modes.

The correspondence between semiclassical Hamiltonians of the form (9) and full quantum Hamiltonians like (1) has been extensively studied in quantum optics $[11,12,13]$. In this field such Hamiltonians model the interaction between quantised electromagnetic readiation and the stationary states of an atom or molecule. The mathematical approaches used focus on Floquet states, which allow the correspondence to be made precise in the infinite phonon number limit. We will use a simpler perturbative approach which, while limited to weak coupling, highlights the difference between the two methods in the case of low phonon number.

\section{Perturbation Theory Analysis}

The assumption of weak coupling means that we can use the first-order results of time-dependent perturbation theory. We shall first state the general results for the 
time-dependence of the electronic energy for the quantum and semiclassical case. We then consider low and high electronic temperature regimes in sections 5.1 and 5.2.

For the quantum case we wish to determine the change in the electronic energy with time,

$$
\begin{aligned}
\Delta E_{\mathrm{e}}(t) & :=\operatorname{Tr}\left\{\hat{\rho}(t) \hat{H}_{\mathrm{e}}\right\}-\operatorname{Tr}\left\{\hat{\rho} \hat{H}_{\mathrm{e}}\right\} \\
& =\operatorname{Tr}\left\{\hat{U}(t) \hat{\rho}(\hat{U}(t))^{+} \hat{H}_{\mathrm{e}}\right\}-\operatorname{Tr}\left\{\hat{\rho} \hat{H}_{\mathrm{e}}\right\}
\end{aligned}
$$

where $\hat{U}(t)$ is the evolution operator for the full quantum system, $\hat{\rho}$ is given by (3) and the trace is over electronic and ionic states. From perturbation theory we obtain a simple picture based on energy and momentum conservation. Consider the scattering of a single electron resulting in some change of momentum q. This change must then correspond to the absorption of a phonon with momentum $\mathbf{q}$ or the emission of one with momentum $\mathbf{- q}$. With this exchange of momentum also comes an absorption or emission of energy given by $\hbar \omega_{\lambda}(\mathbf{q})$ and $\hbar \omega_{\lambda}(-\mathbf{q})$ respectively. Calculating the result explicitly we obtain

$\Delta E_{\mathrm{e}}(t)=\frac{2 \pi t}{\hbar} \sum_{\mathbf{q}, \lambda} \hbar \omega_{\lambda}(\mathbf{q})\left\{\left\langle N_{\mathbf{q}, \lambda}\right\rangle \mathfrak{A}\left(\omega_{\lambda}(\mathbf{q})\right)-\left(\left\langle N_{\mathbf{q}, \lambda}\right\rangle+1\right) \mathfrak{E}\left(\omega_{\lambda}(\mathbf{q})\right)\right\}$.

The full derivation is given in Appendix A. The change of the energy of the electron system is therefore linear with time with a rate set by the absorption (first term of sum) and emission (second term of sum) to each phonon mode. $\mathfrak{A}\left(\omega_{\lambda}(\mathbf{q})\right)$ is an indication of the energy the electrons absorb from the phonon of wavenumber $\mathbf{q}$ and polarisation $\lambda$ and is given by

$\mathfrak{A}\left(\omega_{\lambda}(\mathbf{q})\right)=\int \mathrm{d} E f\left(E-\frac{\hbar \omega_{\lambda}(\mathbf{q})}{2}\right)\left(1-f\left(E+\frac{\hbar \omega_{\lambda}(\mathbf{q})}{2}\right)\right) A\left(E, \omega_{\lambda}(\mathbf{q})\right)$,

where $f(E)$ is the Fermi-Dirac distribution function at electronic temperature $T_{\mathrm{e}}$. The dependence of $f(E)$ on temperature and chemical potential has been suppressed for simplicity. The factor $f\left(E-\frac{\hbar \omega_{\lambda}(\mathbf{q})}{2}\right)$ is the occupation of the electron state scattered from and $\left(1-f\left(E+\frac{\hbar \omega_{\lambda}(\mathbf{q})}{2}\right)\right)$ is one minus the occupation of the electron state scattered to. $\mathfrak{E}\left(\omega_{\lambda}(\mathbf{q})\right)$ is a similar term for the energy the electrons emit to the phonon mode $(\mathbf{q}, \lambda)$,

$\mathfrak{E}\left(\omega_{\lambda}(\mathbf{q})\right)=\int \mathrm{d} E f\left(E+\frac{\hbar \omega_{\lambda}(\mathbf{q})}{2}\right)\left(1-f\left(E-\frac{\hbar \omega_{\lambda}(\mathbf{q})}{2}\right)\right) A\left(E, \omega_{\lambda}(\mathbf{q})\right)$.

The term $A\left(E, \omega_{\lambda}(\mathbf{q})\right)$, which appears in both (12) and (13), is the scattering matrix element averaged over the Fermi surface as follows:

$A\left(E, \omega_{\lambda}(\mathbf{q})\right)=\int_{S_{E+}} \frac{\mathrm{d}^{2} \mathbf{k}^{\prime}}{\left|\nabla \varepsilon_{\mathbf{k}^{\prime}}\right|} \int_{S_{E-}} \frac{\mathrm{d}^{2} \mathbf{k}}{\left|\nabla \varepsilon_{\mathbf{k}}\right|}\left|g_{\lambda}\left(\mathbf{k}, \mathbf{k}^{\prime}\right)\right|^{2} \delta\left(\mathbf{k}-\mathbf{k}^{\prime}-\tilde{\mathbf{k}}-\mathbf{q}\right)$,

where $S_{E+}$ and $S_{E-}$ are constant energy surfaces in electronic k-space defined as

$$
S_{E \pm}=\left\{\mathbf{k} \mid \varepsilon_{\mathbf{k}}=E \pm \frac{\hbar \omega_{\lambda}(\mathbf{q})}{2}\right\}
$$

For the semiclassical case the relevant energy corresponding to (10) is given by

$$
\Delta E_{\mathrm{e}}^{\mathrm{sc}}(t):=\operatorname{Tr}_{\mathrm{e}}\left\{\hat{U}^{\mathrm{sc}}(t) \hat{\rho}_{\mathrm{e}}\left(\hat{U}^{\mathrm{sc}}(t)\right)^{+} \hat{H}_{\mathrm{e}}\right\}-\operatorname{Tr}_{\mathrm{e}}\left\{\hat{\rho}_{\mathrm{e}} \hat{H}_{\mathrm{e}}\right\}
$$


where the trace is over the electronic states and $\hat{U}^{\mathrm{sc}}(t)$ is the semiclassical evolution operator. Evaluating (15) within first-order perturbation theory we obtain

$\Delta E_{\mathrm{e}}^{\mathrm{sc}}(t)=\frac{2 \pi t}{\hbar} \sum_{\mathbf{q}, \lambda} \hbar \omega_{\lambda}(\mathbf{q})\left\{\left\langle N_{\mathbf{q}, \lambda}\right\rangle \mathfrak{A}\left(\omega_{\lambda}(\mathbf{q})\right)-\left\langle N_{\mathbf{q}, \lambda}\right\rangle \mathfrak{E}\left(\omega_{\lambda}(\mathbf{q})\right)\right\}$,

where $\mathfrak{A}\left(\omega_{\lambda}(\mathbf{q})\right)$ and $\mathfrak{E}\left(\omega_{\lambda}(\mathbf{q})\right)$ are as given by (12) and (13) respectively. The full derivation can be found in Appendix B.

Comparisons between the energy exchange in the semiclassical approximation (16) and the full quantum mechanical result (11) show strong similarities, the only difference being a " $+1 "$ in the emission term. If we separate the emission term in to parts corresponding to the spontaneous and stimulated emission,

$$
\begin{aligned}
\Delta E_{\mathrm{e}}(t)= & \frac{2 \pi t}{\hbar} \sum_{\mathbf{q}, \lambda} \hbar \omega_{\lambda}(\mathbf{q})\left\{\left\langle N_{\mathbf{q}, \lambda}\right\rangle \mathfrak{A}\left(\omega_{\lambda}(\mathbf{q})\right)-\left\langle N_{\mathbf{q}, \lambda}\right\rangle \mathfrak{E}\left(\omega_{\lambda}(\mathbf{q})\right)-\mathfrak{E}\left(\omega_{\lambda}(\mathbf{q})\right)\right\} \\
= & \frac{2 \pi t}{\hbar} \sum_{\mathbf{q}, \lambda} \hbar \omega_{\lambda}(\mathbf{q})\{\text { Stimulated Absorption Term } \\
& \quad-\text { Stimulated Emission Term - Spontaneous Emission Term }\},
\end{aligned}
$$

it is clear that the semiclassical approximation (and so equivalently the Ehrenfest approximation) fails when the spontaneous emission term is relevant for the correct evolution of the electronic energy. In retrospect such a failure is to be expected because the spontaneous emission term is an inherently quantum mechanical feature arising from the commutation relation between the annihilation and creation operators of the phonons.

In order to ascertain the physical situations in which spontaneous emission of phonons is important we must evaluate $\left(\mathfrak{A}\left(\omega_{\lambda}(\mathbf{q})-\mathfrak{E}\left(\omega_{\lambda}(\mathbf{q})\right)\right.\right.$ and $\mathfrak{E}\left(\omega_{\lambda}(\mathbf{q})\right)$ for a range of different physical parameters. The former enters in to terms where electron behaviour is stimulated while the latter is involved in the spontaneous emission term. We shall find that in both cases of low and high electronic temperature the semiclassical approximation is only accurate when the temperature of the oscillator system is much larger than the temperature of the electronic system.

\subsection{Low Electronic Temperatures}

We first consider low electronic temperatures. In such cases the energies of those electrons which absorb a phonon with energy $\omega_{\lambda}(\mathbf{q})$ are restricted to lie within $\hbar \omega_{\lambda}(\mathbf{q})$ below the Fermi energy. This suggests we can make the following assumptions in the case of low temperatures:

(i) We assume that for the energies of electrons which take part in scattering processes $A\left(E, \omega_{\lambda}(\mathbf{q})\right)$ is constant. This assumption becomes increasingly invalid as we consider higher phonon energies as the range of electronic energies involved increases.

(ii) We assume that electron states at the top and bottom of the band are not involved in phonon emission and absorption processes. Again this is increasingly invalid at higher phonon energies. 
We then find (see Appendix C)

$$
\begin{aligned}
& \left(\mathfrak{A}\left(\omega_{\lambda}(\mathbf{q})\right)-\mathfrak{E}\left(\omega_{\lambda}(\mathbf{q})\right)\right)=A\left(E_{0}, \omega_{\lambda}(\mathbf{q})\right) \hbar \omega_{\lambda}(\mathbf{q}), \\
& \mathfrak{E}\left(\omega_{\lambda}(\mathbf{q})\right)=A\left(E_{0}, \omega_{\lambda}(\mathbf{q})\right) \hbar \omega_{\lambda}(\mathbf{q}) \frac{1}{\exp \left(\frac{\hbar \omega_{\lambda}(\mathbf{q})}{k_{\mathrm{B}} T_{\mathrm{e}}}\right)-1},
\end{aligned}
$$

where $A\left(E_{0}, \omega_{\lambda}(\mathbf{q})\right)$ is the single value of $A$ over the relevant energy interval. Inserting these results in to (11) and (16)

$$
\begin{aligned}
& \Delta E_{\mathrm{e}}(t)=\frac{2 \pi t}{\hbar} \sum_{\mathbf{q}, \lambda}\left(\hbar \omega_{\lambda}(\mathbf{q})\right)^{2} A\left(E_{0}, \omega_{\lambda}(\mathbf{q})\right)\left\{\left\langle N_{\mathbf{q}, \lambda}\right\rangle-\frac{1}{\exp \left(\frac{\hbar \omega_{\lambda}(\mathbf{q})}{k_{\mathrm{B}} T_{\mathrm{e}}}\right)-1}\right\}, \\
& \Delta E_{\mathrm{e}}^{\mathrm{sc}}(t)=\frac{2 \pi t}{\hbar} \sum_{\mathbf{q}, \lambda}\left(\hbar \omega_{\lambda}(\mathbf{q})\right)^{2} A\left(E_{0}, \omega_{\lambda}(\mathbf{q})\right)\left\langle N_{\mathbf{q}, \lambda}\right\rangle .
\end{aligned}
$$

So, in the low electronic temperature limit, the criterion for the success of the semiclassical approximation is given by

$$
\left\langle N_{\mathbf{q}, \lambda}\right\rangle=\frac{1}{\exp \left(\frac{\hbar \omega_{\lambda}(\mathbf{q})}{k_{\mathrm{B}} T_{\mathrm{osc}}}\right)-1} \gg \frac{1}{\exp \left(\frac{\hbar \omega_{\lambda}(\mathbf{q})}{k_{\mathrm{B}} T_{\mathrm{e}}}\right)-1},
$$

where we have noted (3) results in modes occupied according to the Bose-Einstein distribution. Thus we require that $T_{\mathrm{osc}} \gg T_{\mathrm{e}}$, although it is probably better to use the criterion of (22) in any quantitative analysis.

Comparisons between (20) and (21) also make it clear that the semiclassical approximation fails to give an equilibrium between the electron and ion systems. So, although we can use (6) to ensure that the initial temperature of the ions in the Ehrenfest simulation is the same as that of the electrons, failures inherent in the Ehrenfest approximation means we will still see an energy flow to the electrons.

\subsection{High Electronic Temperatures}

We now consider such high electronic temperatures that all the electronic states in the band are approximately equally occupied. This means that the effect of the FermiDirac functions can be taken outside of the integral in (12) and (13).

We find that (details are given in Appendix D)

$$
\begin{aligned}
& \left(\mathfrak { A } \left(\omega_{\lambda}(\mathbf{q})-\mathfrak{E}\left(\omega_{\lambda}(\mathbf{q})\right)=\frac{1}{4} \frac{\hbar \omega_{\lambda}(\mathbf{q})}{k_{\mathrm{B}} T_{\mathrm{e}}} \int \mathrm{d} E A\left(E, \omega_{\lambda}(\mathbf{q})\right),\right.\right. \\
& \mathfrak{E}\left(\omega_{\lambda}(\mathbf{q})\right)=\frac{1}{4}\left(1-\frac{\hbar \omega_{\lambda}(\mathbf{q})}{2 k_{\mathrm{B}} T_{\mathrm{e}}}\right) \int \mathrm{d} E A\left(E, \omega_{\lambda}(\mathbf{q})\right) .
\end{aligned}
$$

By (11) and (16) we have for the energy exchange between the electrons and ions

$$
\begin{aligned}
& \Delta E(t)=\frac{2 \pi t}{\hbar} \sum_{\mathbf{q}, \lambda} \frac{1}{4} \hbar \omega_{\lambda}(\mathbf{q})\left\{\left(\left\langle N_{\mathbf{q}, \lambda}\right\rangle+\frac{1}{2}\right) \frac{\hbar \omega_{\lambda}(\mathbf{q})}{k_{\mathrm{B}} T_{\mathrm{e}}}-1\right\} \int \mathrm{d} E A\left(E, \omega_{\lambda}(\mathbf{q})\right), \\
& \Delta E^{\mathrm{sc}}(t)=\frac{2 \pi t}{\hbar} \sum_{\mathbf{q}, \lambda} \frac{1}{4} \hbar \omega_{\lambda}(\mathbf{q})\left\{\left\langle N_{\mathbf{q}, \lambda}\right\rangle \frac{\hbar \omega_{\lambda}(\mathbf{q})}{k_{\mathrm{B}} T_{\mathrm{e}}}\right\} \int \mathrm{d} E A\left(E, \omega_{\lambda}(\mathbf{q})\right) .
\end{aligned}
$$

We can use (25) and (26) to determine the following criterion for the success of the Ehrenfest approximation

$$
\left\langle N_{\mathbf{q}, \lambda}\right\rangle \frac{\hbar \omega_{\lambda}(\mathbf{q})}{k_{\mathrm{B}} T_{\mathrm{e}}} \gg 1-\frac{\hbar \omega_{\lambda}(\mathbf{q})}{2 k_{\mathrm{B}} T_{\mathrm{e}}} .
$$


Ignoring the second term on the right hand side due to its relatively small size and using the fact that in the high temperature limit we have

$$
\frac{\hbar \omega_{\lambda}(\mathbf{q})}{k_{\mathrm{B}} T_{\mathrm{e}}} \approx \exp \left(\frac{\hbar \omega_{\lambda}(\mathbf{q})}{k_{\mathrm{B}} T_{\mathrm{e}}}\right)-1
$$

and so we can write $(27)$ as

$$
\left\langle N_{\mathbf{q}, \lambda}\right\rangle=\frac{1}{\exp \left(\frac{\hbar \omega_{\lambda}(\mathbf{q})}{k_{\mathrm{B}} T_{\mathrm{osc}}}\right)-1} \gg \frac{1}{\exp \left(\frac{\hbar \omega_{\lambda}(\mathbf{q})}{k_{\mathrm{B}} T_{\mathrm{e}}}\right)-1},
$$

which is the same criterion as for the low temperature case.

The central difference between (25) and (26) is that the former contains a term that is independent of the electronic temperature. This term comes to dominate as the electronic temperature increases resulting in a flow of energy from the electrons to the ions. This is to be expected, energy flows from hot electrons to relatively cold ions. The contrast with (26) highlights that this energy flow is only possible with the inclusion of quantum mechanical behaviour of the oscillator system. The possibility of spontaneous emission of phonons is entirely responsible for the flow of energy back in to the ions.

\section{Results for a Simple Model}

In this section we provide support for the analytical results of previous sections by considering a simple model system. We first state the Hamiltonian of the system and then derive analytical results equivalent to those of section 5. Finally we present exact numerical results and compare them with the analytical formulae.

We consider electrons on one-dimensional chain of $L$ sites subject to periodic boundary conditions with nearest neighbour hopping of strength $b$ coupled to a single oscillator of mass $m$ and angular frequency $\omega$ at a single site. We couple to a single oscillator as opposed to multiple oscillators to ease our numerical analysis. The Hamiltonian of the system is

$$
\begin{aligned}
\hat{H}= & b\left(\sum_{i=0}^{L-2}\left(\hat{d}_{i}^{+} \hat{d}_{i+1}+\hat{d}_{i+1}^{+} \hat{d}_{i}\right)+\hat{d}_{L-1}^{+} \hat{d}_{0}+\hat{d}_{0}^{+} \hat{d}_{L-1}\right) \\
& +\left(\frac{\hat{p}^{2}}{2 m}+\frac{1}{2} m \omega^{2} \hat{x}^{2}\right)+\lambda \hat{d}_{0}^{+} \hat{d}_{0} \hat{x}
\end{aligned}
$$

Here $\hat{d}_{i}^{+}$and $\hat{d}_{i}$ are the creation and annihilation operators for an electron on site $i ; \hat{p}$ and $\hat{x}$ are the momentum and position operators for the oscillator and $\lambda$ is an indication of the strength of the electron-phonon coupling. The translational symmetry of the electron system means we can attach the oscillator to any site without affecting the result.

Let $|\mathbf{K} N\rangle$ be an eigenstate of the uncoupled system where the oscillator has $N$ phonons and the electrons are in an eigenstate $\mathbf{K}$ of the uncoupled electron system. The initial state of the system is chosen to be

$$
\hat{\rho}=\sum_{\mathbf{K}} \frac{1}{Z_{\mathrm{e}}} \exp \left(\frac{E_{\mathbf{K}}}{k_{\mathrm{B}} T_{\mathrm{e}}}\right)|\mathbf{K} N\rangle\langle\mathbf{K} N|,
$$

where we have used the same notation as (3). For simplicity we consider an oscillator with a given initial phonon number, $N$, rather than consider a finite temperature $T_{\text {osc }}$. 
Using the methods detailed in sections 3 and 4 we are able to determine the Ehrenfest and semiclassical approximations of (28) with initial conditions (29). In the Ehrenfest approximation the evolution of the system is given by the two coupled equations

$$
\begin{aligned}
& \mathrm{i} \hbar \dot{\hat{\rho}}_{\mathrm{e}}(t)=\left[b\left(\sum_{i=0}^{L-2}\left(\hat{d}_{i}^{+} \hat{d}_{i+1}+\hat{d}_{i+1}^{+} \hat{d}_{i}\right)+\hat{d}_{L-1}^{+} \hat{d}_{0}+\hat{d}_{0}^{+} \hat{d}_{L-1}\right)+\lambda \hat{d}_{0}^{+} \hat{d}_{0} R(t), \hat{\rho}_{\mathrm{e}}(t)\right] \\
& m \ddot{R}(t)=-m \omega^{2} R(t)-\operatorname{Tr}\left\{\hat{\rho}_{\mathrm{e}}(t)\left(\lambda \hat{d}_{0}^{+} \hat{d}_{0}\right)\right\} .
\end{aligned}
$$

The coupled oscillator is now modelled classically and its position is denoted by $R(t)$. In order to set the initial amplitude of the oscillator we use the 1 dimensional quantum to classical correspondence given by (7). The initial electronic density matrix is found by tracing out the oscillator state from (29). As in section 4 we derive the semiclassical Hamiltonian from the Ehrenfest approximation by assuming that the weak electronphonon coupling does not influence the classical motion of the coupled oscillator. Therefore its classical motion is given by $R(t)=A \cos \omega t$ leading to the semiclassical Hamiltonian

$$
\begin{aligned}
\hat{H}^{\mathrm{sc}}(t)= & b\left(\sum_{i=0}^{L-2}\left(\hat{d}_{i}^{+} \hat{d}_{i+1}+\hat{d}_{i+1}^{+} \hat{d}_{i}\right)+\hat{d}_{L-1}^{+} \hat{d}_{0}+\hat{d}_{0}^{+} \hat{d}_{l-1}\right) \\
& +\lambda A \hat{d}_{0}^{+} \hat{d}_{0}\left(\frac{\mathrm{e}^{\mathrm{i} \omega t}+\mathrm{e}^{-\mathrm{i} \omega t}}{2}\right) .
\end{aligned}
$$

We now apply the techniques of first-order time-dependent perturbation theory to determine the evolution of the fully quantum mechanical system governed by (28) and the semiclassical system governed by (32). We can then obtain the change of the electronic energy as defined by (10) and (15). For the fully quantum system we find

$$
\Delta E_{\mathrm{e}}=\frac{2 \pi t}{\hbar} \hbar \omega\{N \mathfrak{A}-(N+1) \mathfrak{E}\},
$$

where the absorption and emission coefficients are given by

$$
\begin{aligned}
& \mathfrak{A}=\lambda^{2} \frac{\hbar}{2 m \omega} \int_{B} \mathrm{~d} E n\left(E-\frac{\hbar \omega}{2}\right) n\left(E+\frac{\hbar \omega}{2}\right) f\left(E-\frac{\hbar \omega}{2}\right)\left(1-f\left(E+\frac{\hbar \omega}{2}\right)\right), \\
& \mathfrak{E}=\lambda^{2} \frac{\hbar}{2 m \omega} \int_{B} \mathrm{~d} E n\left(E+\frac{\hbar \omega}{2}\right) n\left(E-\frac{\hbar \omega}{2}\right) f\left(E+\frac{\hbar \omega}{2}\right)\left(1-f\left(E-\frac{\hbar \omega}{2}\right)\right) .
\end{aligned}
$$

Here $n(E)$ denotes the density of states per site of the uncoupled electron system, $f(E)$ is the Fermi-Dirac distribution at temperature $T_{\mathrm{e}}$ and the range of integration is given by $B=\left\{E \mid-2 b+\frac{\hbar \omega}{2} \leq E \leq 2 b-\frac{\hbar \omega}{2}\right\}$. For the semiclassical system we have

$$
\Delta E_{\mathrm{e}}^{\mathrm{sc}}=\frac{2 \pi t}{\hbar} \hbar \omega\{N \mathfrak{A}-N \mathfrak{E}\},
$$

using the relation between oscillator amplitude and phonon number given by (7). As we saw in section 5 for the more complex multiple oscillator system, the semiclassical approximation fails when the spontaneous emission term is relevant.

We can go on to perform an analysis of the low and high temperature limits of (34) and (35) using the methods of sections 5.1 and 5.2. An analysis of these results is required to confirm that the simple picture of section 5 is valid. In the case of low temperatures we follow the approach of section 5.1 and assume the density of states 
is flat for scattered electrons and that the states at the top and bottom of the band are not involved. We then obtain

$\Delta E_{\mathrm{e}}=\frac{2 \pi t}{\hbar} \lambda^{2} \frac{\hbar}{2 m \omega}(\hbar \omega)^{2}\left(n\left(E_{0}\right)\right)^{2}\left(N-\frac{1}{\exp \left(\frac{\hbar \omega}{k_{\mathrm{B}} T_{\mathrm{e}}}\right)-1},\right)$
$\Delta E_{\mathrm{e}}^{\mathrm{sc}}=\frac{2 \pi t}{\hbar} \frac{\hbar}{2 m \omega} \lambda^{2}(\hbar \omega)^{2}\left(n\left(E_{0}\right)\right)^{2} N$

where $n\left(E_{0}\right)$ is the single value of the density of states over the relevant energies. (See Appendix $\mathrm{C}$ for a full derivation.) In comparing the energy changes given by full quantum system and the semiclassical approximation we obtain the following ratio

$$
\frac{\Delta E_{\mathrm{e}}}{\Delta E_{\mathrm{e}}^{\mathrm{sc}}}=1-\frac{1}{N\left(\exp \left(\frac{\hbar \omega}{k_{\mathrm{B}} T_{\mathrm{e}}}\right)-1\right)} .
$$

For sufficiently high temperatures electronic states across the whole band are equally occupied and we find

$\begin{aligned} & \Delta E_{\mathrm{e}}=\frac{2 \pi t}{\hbar} \hbar \omega \frac{\hbar}{2 m \omega} \lambda^{2} \frac{1}{4}\left(\left(N+\frac{1}{2}\right) \frac{\hbar \omega}{k_{\mathrm{B}} T_{\mathrm{e}}}-1\right) \int_{B} \mathrm{~d} E n\left(E-\frac{\hbar \omega}{2}\right) n\left(E+\frac{\hbar \omega}{2}\right)( \\ & \Delta E_{\mathrm{e}}^{\mathrm{sc}}=\frac{2 \pi t}{\hbar} \hbar \omega \frac{\hbar}{2 m \omega} \lambda^{2} \frac{1}{4}\left(N \frac{\hbar \omega}{k_{\mathrm{B}} T_{\mathrm{e}}}\right) \int_{B} \mathrm{~d} E n\left(E-\frac{\hbar \omega}{2}\right) n\left(E+\frac{\hbar \omega}{2}\right)\end{aligned}$

For high temperatures the ratio of the two energy changes is given by

$$
\frac{\Delta E_{\mathrm{e}}}{\Delta E_{\mathrm{e}}^{\mathrm{sc}}}=1+\frac{1}{2 N}-\frac{k_{\mathrm{B}} T_{\mathrm{e}}}{N \hbar \omega}
$$

We remark on the similarity between the ratios for the high and low temperature cases. To see this we can, for high temperatures, use the Taylor expansion of $\exp (x)$ about zero we can rewrite $(42)$ as

$$
\frac{\Delta E_{\mathrm{e}}}{\Delta E_{\mathrm{e}}^{\mathrm{sc}}}=1+\frac{1}{2 N}-\frac{1}{N\left(\exp \left(\frac{\hbar \omega}{k_{\mathrm{B}} T_{\mathrm{e}}}\right)-1\right)},
$$

This completes our review of analytical results for the model Hamiltonian (28).

In the rest of this section we shall confirm the above formulae numerically. We performed three different numerical procedures:

(i) An Ehrenfest simulation using (30) and (31).

(ii) The occupations of the system's eigenstates calculated using a first-order timedependent perturbation theory analysis of the semiclassical result (32) (before the long time limit that is taken to give Fermi's golden rule).

(iii) A numerical integration of (34) and (35) using an adaptive Simpson's rule. The Fermi's golden rule result (33) is then used to give the electronic energy change for the full quantum system $\ddagger$. To determine the density of states we assume the electron system is infinitely large which gives (see figure 1)

$$
n(E)= \begin{cases}\frac{1}{2 b \pi}\left(1-\left(\frac{E}{2 b}\right)^{2}\right)^{-\frac{1}{2}} & \text { when }-2 b \leq E \leq 2 b \\ 0 & \text { otherwise. }\end{cases}
$$

$\ddagger$ There are issues regarding the time scales over which Fermi's golden rule is applicable to finite systems [14]. We have found that our system is sufficiently large to compare with Fermi's golden rule over the times considered. 
Table 1. A table of the parameters used in the numerical procedures.

\begin{tabular}{|c|c|}
\hline Parameter & Value \\
\hline$b$ & $2 \hbar \omega$ \\
$\lambda$ & $0.001 \hbar \omega \sqrt{\frac{m \omega}{\hbar}}$ \\
$L$ & 200 \\
\hline
\end{tabular}

Figure 1. The density of states of the uncoupled electron system and the FermiDirac distribution for the three temperatures used in figures 2-4

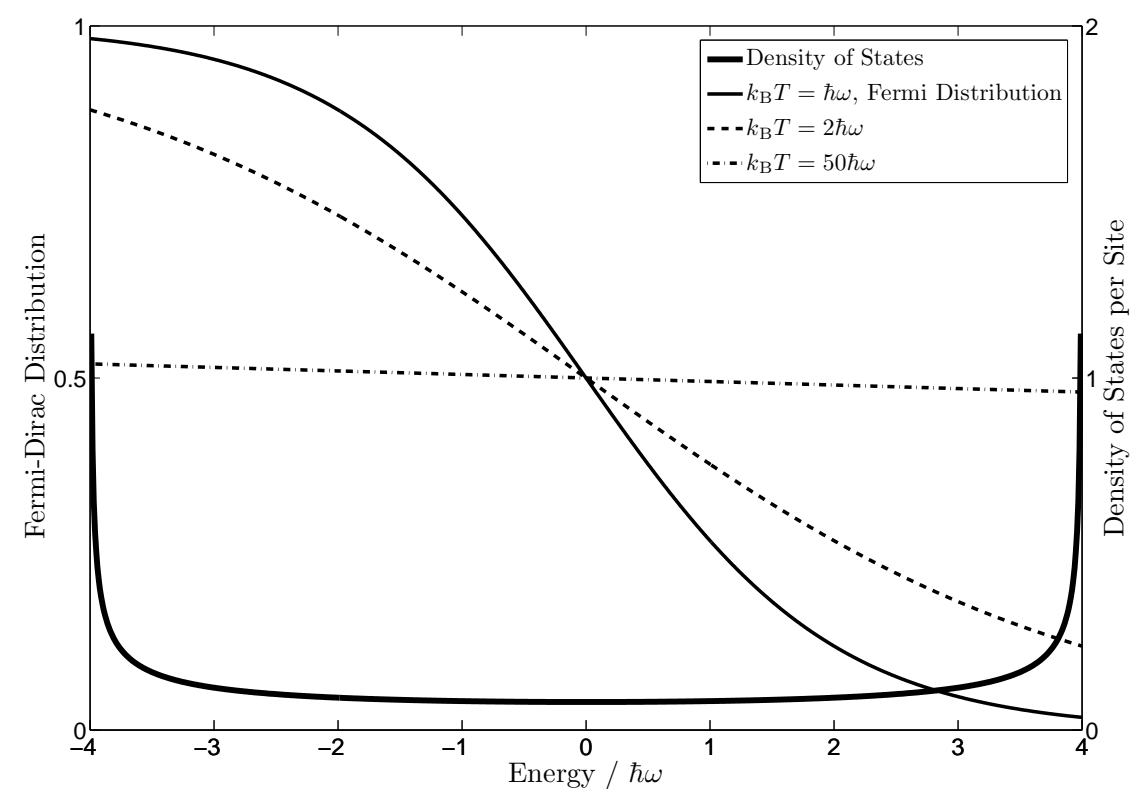

We have not undertaken a full quantum mechanical simulation because the presence of a quantum mechanical oscillator introduces phonon mediated electron-electron interactions. Therefore to correctly describe this system would require storing twoparticle density matrices, three-particle density matrices, etc.. The system parameters used are given in table 1 and are chosen specifically to ensure we are in the region of weak coupling. We start the density matrix at three different temperatures, the corresponding Fermi factors are shown in figure 1. The Fermi factors of the lowest (highest) temperature is such that it satisfies conditions required for equation (39) (equation (42)). For each temperature we consider a range of initial phonon numbers to highlight when the Ehrenfest approximation is accurate. The system is evolved from $\omega t_{0}=0$ to $\omega t_{\mathrm{f}}=40$.

The time-dependence of the quantities

$$
\frac{\Delta E_{\mathrm{e}}(t)}{\Delta E_{\mathrm{e}}^{\mathrm{ehr}}\left(t_{\mathrm{f}}\right)}, \frac{\Delta E_{\mathrm{e}}^{\mathrm{sc}}(t)}{\Delta E_{\mathrm{e}}^{\mathrm{ehr}}\left(t_{\mathrm{f}}\right)} \text { and } \frac{\Delta E_{\mathrm{e}}^{\mathrm{ehr}}(t)}{\Delta E_{\mathrm{e}}^{\mathrm{ehr}}\left(t_{\mathrm{f}}\right)},
$$

are shown in figures 2-4. Here $\Delta E_{\mathrm{e}}^{\mathrm{ehr}}$ denotes the evolution of the electronic energy 
Figure 2. The evolution of the electronic energy from our three numerical procedures for initial temperature $k_{\mathrm{B}} T_{\mathrm{e}}=\hbar \omega$ over a range of initial phonon numbers. The plots are scaled so that the Ehrenfest result ends at 1 for all phonon numbers. The semiclassical and Ehrenfest results are indistiguishable. As the phonon number increases the Ehrenfest and semiclassical approximations near the fully quantum mechanical Fermi's golden rule result.

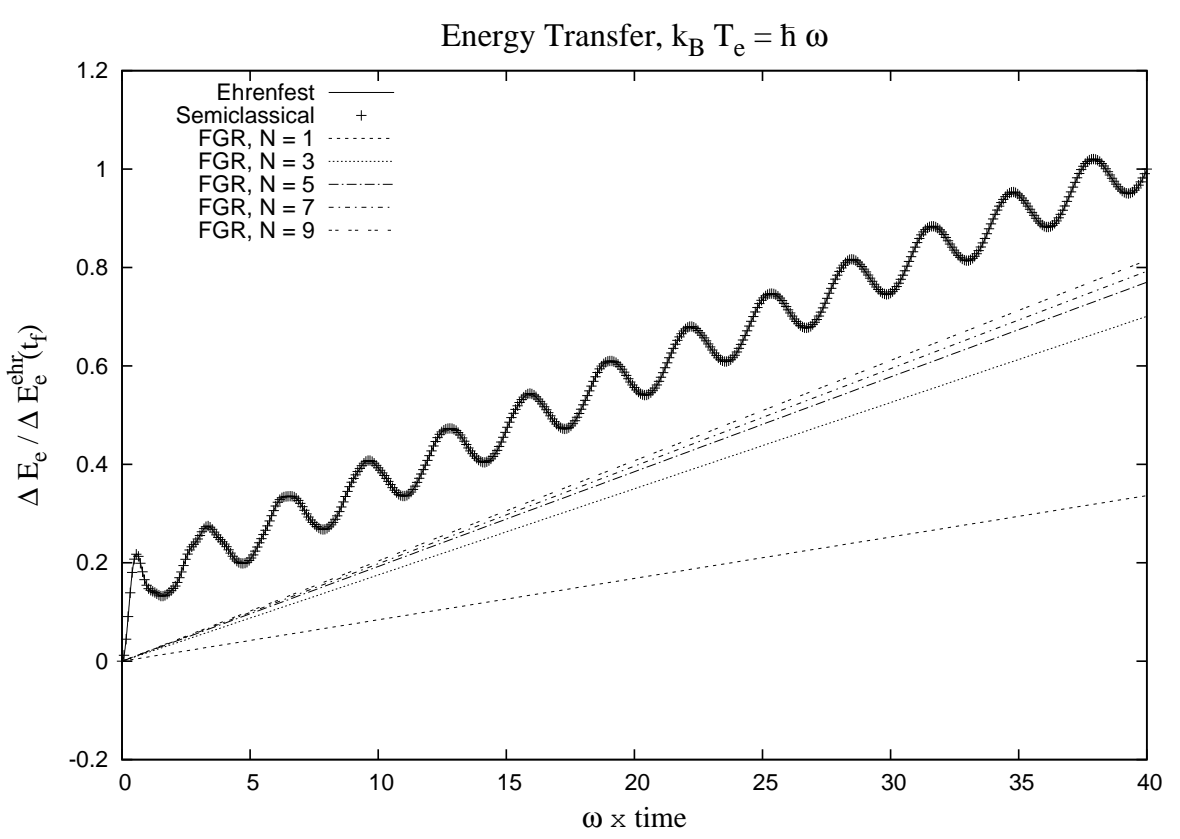

under the Ehrenfest equations (30) and (31). By dividing by the Ehrenfest energy at $t_{\mathrm{f}}$ all of the Ehrenfest results to coincide. We first note that in these plots the Ehrenfest results and the semiclassical results are identical, a conclusion borne out by examination of individual datapoints. This vindicates our claim that for weak coupling a semiclassical Hamiltonian gives the same electronic energy evolution as a full Ehrenfest simulation. It also provides a simple explanation for why all the Ehrenfest results coincide when rescaled since, as seen in (36), the initial amplitude simply acts as a scaling factor which is cancelled by the division of $\Delta E_{\mathrm{e}}^{\mathrm{ehr}}\left(t_{\mathrm{f}}\right)$.

For all three temperatures plotted we see that, as expected, the approximate results are more accurate the higher the initial phonon number. The gradual convergence of the Fermi's golden rule result and the approximations is clear in all three plots. For all three temperatures the semiclassical and Ehrenfest behaviour show a rapid initial increase in energy resulting in the plots appearing "shifted." This is a result of choosing the coupled oscillator to be initially displaced so that $\hat{\rho}_{\mathrm{e}}$ is not a sum of eigenstates of $\hat{H}^{\mathrm{sc}}(0)$. Essentially the initial Hamiltonian can be viewed as sudden perturbation and this causes the rapid change. This issue can be resolved by starting the oscillator at $R(0)=0$ with a set initial velocity $\dot{R}=0$ but such effort is generally unnecessary as the rate of increase can easily be discerned from the plots and the "shift" becomes increasingly irrelevant as time goes on.

The accuracy of the approximations can be seen in more detail in column three 
The Ehrenfest approximation for electrons and phonons

Figure 3. The evolution of the electronic energy from our three numerical procedures for initial temperature $k_{\mathrm{B}} T_{\mathrm{e}}=2 \hbar \omega$.

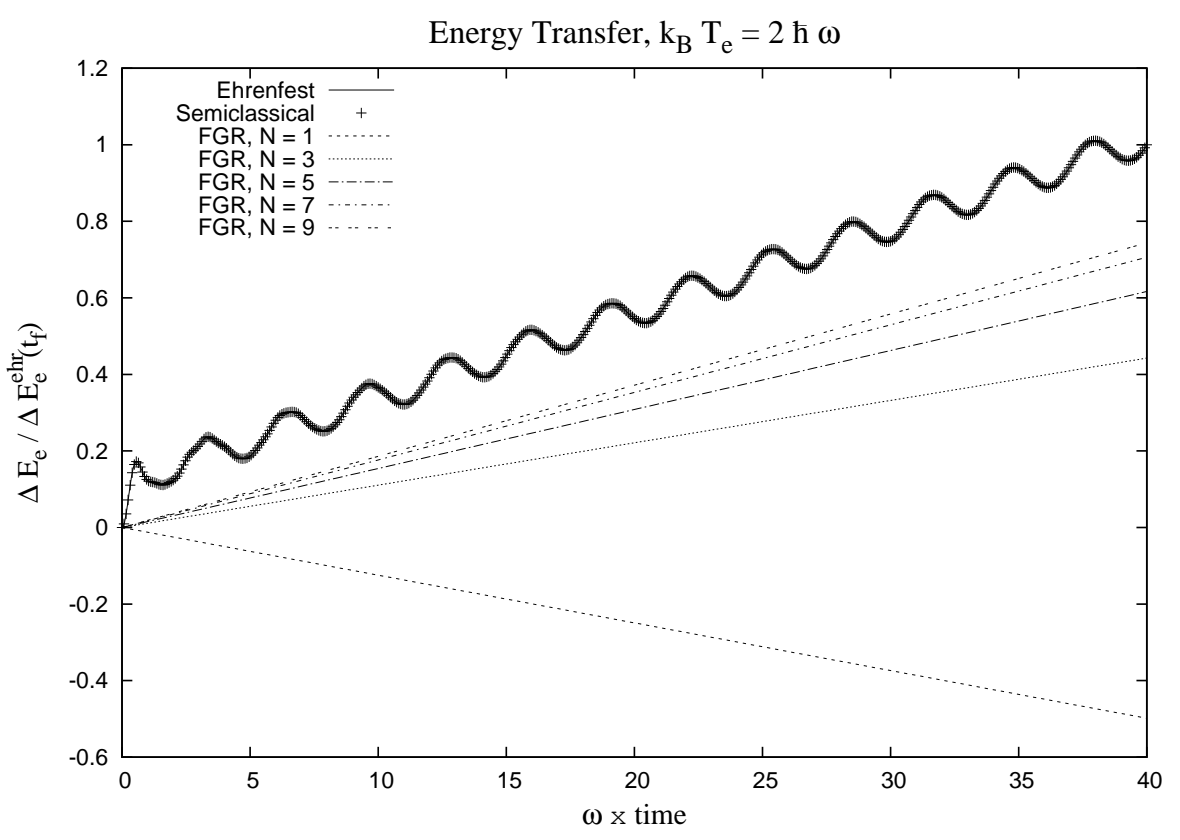

Figure 4. A plot of the evolution of the electronic energy from our three numerical procedures for initial temperature $k_{\mathrm{B}} T_{\mathrm{e}}=50 \hbar \omega$.

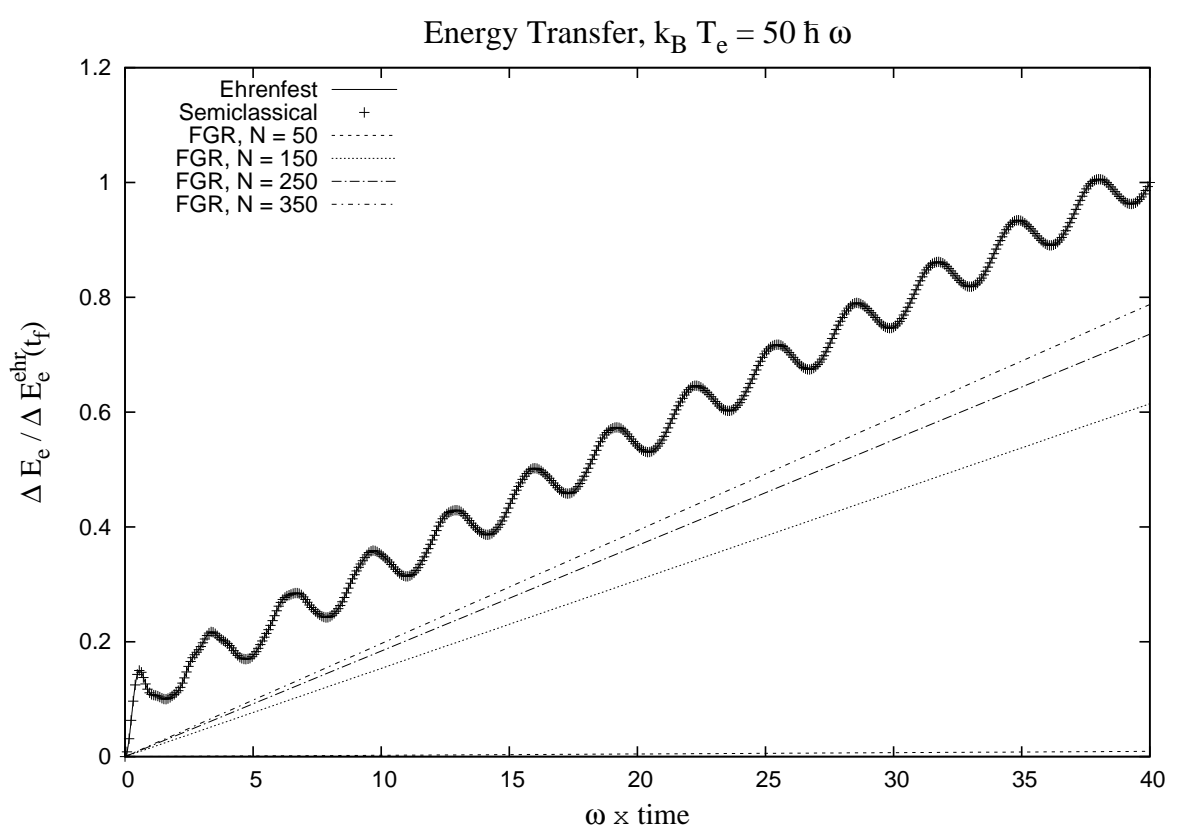


Table 2. A table of the ratio $\Delta E_{\mathrm{e}} / \Delta E_{\mathrm{e}}^{\mathrm{sc}}$ for different temperatures and amplitudes. The closer this ratio to 1 the more accurate the semiclassical and Ehrenfest approximations. We also show the value of this ratio as estimated by the low temperature result (39) and the high temperature result (42).

\begin{tabular}{|c|c|c|c|c|}
\hline$\frac{k_{\mathrm{B}} T_{\mathrm{e}}}{\hbar \omega}$ & $N$ & $\Delta E_{\mathrm{e}} / \Delta E_{\mathrm{e}}^{\mathrm{sc}}$ & Approx Ratio $\left(\right.$ Low $\left.T_{\mathrm{e}}\right)$ & Approx Ratio $\left(\right.$ High $\left.T_{\mathrm{e}}\right)$ \\
\hline 1 & 1 & 0.3874 & 0.4180 & 0.5000 \\
1 & 3 & 0.8066 & 0.8060 & 0.8333 \\
1 & 5 & 0.8862 & 0.8836 & 0.9000 \\
1 & 7 & 0.9120 & 0.9169 & 0.9286 \\
1 & 9 & 0.9375 & 0.9353 & 0.9444 \\
2 & 1 & -0.5544 & -0.5415 & -0.5000 \\
2 & 3 & 0.4921 & 0.4862 & 0.5000 \\
2 & 5 & 0.6852 & 0.6917 & 0.7000 \\
2 & 7 & 0.7847 & 0.7798 & 0.7857 \\
2 & 9 & 0.8265 & 0.8287 & 0.8333 \\
50 & 1 & -48.4900 & -48.5017 & -48.5000 \\
50 & 50 & 0.0100 & 0.0100 & 0.0100 \\
50 & 150 & 0.6704 & 0.6700 & 0.6700 \\
50 & 250 & 0.8024 & 0.8020 & 0.8020 \\
50 & 350 & 0.8590 & 0.8586 & 0.8586 \\
\hline
\end{tabular}

of table 2. For temperature $k_{\mathrm{B}} T_{\mathrm{e}}=\hbar \omega$ the Ehrenfest approximation is $80 \%$ accurate for $N \geq 3$. For the higher temperature $k_{\mathrm{B}} T_{\mathrm{e}}=2 \hbar \omega, 80 \%$ accuracy is only achieved for $N \geq 9$. At the much high temperature $k_{\mathrm{B}} T_{\mathrm{e}}=50 \hbar \omega$, for which the whole band is approximately equally occupied, the Ehrenfest approximation and Fermi's golden rule only agree for very high phonon numbers $N \geq 250$. The inability of the semiclassical Hamiltonian to give a transfer of energy from electrons to ions is made obvious here. For the parameter sets $\left\{k_{\mathrm{B}} T_{\mathrm{e}}=2 \hbar \omega, N=1\right\}$ and $\left\{k_{\mathrm{B}} T_{\mathrm{e}}=50 \hbar \omega, N=1\right\}$ Fermi's golden rule indicates that the rate of electronic energy change should be negative but the Ehrenfest approximation gives a positive rate. Also shown in columns 4 and 5 of table 2 are the estimated energy ratios given by consideration of the low temperature limit (39) and high temperature limit (42). For this simple model both of the estimates prove to be close to the true value for all temperature ranges. This is due to the electronic system's predominantly flat density of states, as seen in figure 1 . As you would expect, the low temperature limit proves to be slightly more accurate for $k_{\mathrm{B}} T_{\mathrm{e}}=\hbar \omega, 2 \hbar \omega$. Furthermore it is equally accurate for $k_{\mathrm{B}} T_{\mathrm{e}}=50 \hbar \omega$ because, as discussed, the two results converge for high temperatures and many phonons.

All the numerical results that we have obtained provide validation for all the analytical work done for this simple model. We can thus have confidence in the more general results of section 5 .

\section{Conclusions}

We have suggested a simple scheme for applying the Ehrenfest approximation to simulations of solids. Assuming an initial density matrix of the form (3) the quantum to classical correspondence given by (6) can be used to set the initial amplitude of 
each mode which can in turn be used to set the initial position and momentum of the classical ions of the Ehrenfest approximation.

Assuming that the change in the potential due to the electrons has a negligible effect on the behaviour of the ions, we analysed the energy flow to the electrons in the Ehrenfest approximation using a simpler semiclassical Hamiltonian (9). We found that when the ion temperature is much higher than that of the electrons, the Ehrenfest approximation will give the correct energy exchange between the two systems.

The Ehrenfest approximation does not encompass several important features of electron-ion coupling. We will not see equilibration between the electrons and ions. Even if the quantum to classical correspondence is used to set up the ions in a state with the same "temperature" as the electrons, we will still see an energy flow to the electron system. Furthermore, if the electron system is hotter than the ion system, the Ehrenfest approximation will give an energy flow in the wrong direction, from the ions to the electrons. These results can be encompassed in the statement that the Ehrenfest approximation only correctly includes the energy flow from ions to electrons and fails when the electron to ion energy exchange is important. This observation has been made before on the basis of numerical observation [3]. In this work we traced this failure to the absence of the quantum mechanical effect of spontaneous phonon emission in the Ehrenfest approximation. The numerical results of section 6 support these conclusions.

Although the initial states we have considered were restricted to the form (3) we suspect that the conclusions of this paper apply more generally. Out heuristic discussion emphasised the importance of the expected phonon number over all other properties of the ion system. It is therefore not unreasonable to extend our rigorous results regarding the Ehrenfest approximation's validity to a general high energy phonon system interacting with a low energy electron system.

The analysis of the Ehrenfest approximation in this paper is incomplete in two important ways. We modelled the ions as a phonon system and we neglected the "mean-field" effect of the electrons on the ions. In particularly violent events, for example at the start of a radiation damage cascade, these assumptions are no longer valid. Phonon-phonon interactions will be relevant so the first assumption is invalid. Furthermore the electrons will undergo a substantial change in state meaning that the force due to the electrons in the ions will also change substantially. This work is therefore applicable only to the later stages of the cascade where the ions behave as a very high temperature oscillator system and the electrons act primarily as a heat sink.

\section{Acknowledgments}

JleP would like to acknowledge the support of the States of Guernsey. DRM was supported by EPSRC grant number EP/C524403.

\section{Appendix A. Derivation of Equation (11)}

We start with the Hamiltonian given by (1) and treat the coupling as a perturbation, denoted by $\hat{V}$, to the Hamiltonian of the uncoupled electrons and ions, denoted by $\hat{H}_{0}$. The eigenstates of $\hat{H}_{0}$ are given by (2). We define the perturbed and unperturbed 
propagators by

$$
\hat{U}(t):=\exp \left(-\frac{\mathrm{i} \hat{H} t}{\hbar}\right) \text { and } \hat{U}_{0}(t):=\exp \left(-\frac{\mathrm{i} \hat{H}_{0} t}{\hbar}\right) .
$$

We wish to determine the change in the electronic energy $\Delta E_{\mathrm{e}}(t)$ as defined by (10) with the system initially described by the density matrix (3).

As the system evolves each eigenstate of the uncoupled system $|\mathbf{K} \boldsymbol{\Phi}\rangle$ will become a superposition of all other $\left|\mathbf{K}^{\prime} \boldsymbol{\Phi}^{\prime}\right\rangle$. Thus we define

$$
|(\mathbf{K} \boldsymbol{\Phi})(t)\rangle:=\sum_{\mathbf{K}^{\prime}, \boldsymbol{\Phi}^{\prime}} C_{\mathbf{K}^{\prime} \boldsymbol{\Phi}^{\prime}, \mathbf{K} \boldsymbol{\Phi}}(t)\left|\mathbf{K}^{\prime} \boldsymbol{\Phi}^{\prime}\right\rangle
$$

where $C_{\mathbf{K}^{\prime} \boldsymbol{\Phi}^{\prime}, \mathbf{K} \boldsymbol{\Phi}}(t)=\left\langle\mathbf{K}^{\prime} \boldsymbol{\Phi}^{\prime}, \hat{U}(t) \mathbf{K} \boldsymbol{\Phi}\right\rangle$. If the system is initially given by (3) then the change in electronic energy is given by

$$
\begin{gathered}
\Delta E_{\mathrm{e}}(t)=\sum_{\mathbf{K}} \frac{1}{Z_{\mathrm{e}}} \exp \left(-\frac{E_{\mathbf{K}}}{k_{\mathrm{B}} T_{\mathrm{e}}}\right) \sum_{\mathbf{\Phi}} \frac{1}{Z_{\mathrm{ion}}} \exp \left(-\frac{W_{\mathbf{\Phi}}}{k_{\mathrm{B}} T_{\mathrm{ion}}}\right) \\
\left(\sum_{\substack{\mathbf{K}^{\prime}, \boldsymbol{\Phi}^{\prime} \\
\mathbf{K}^{\prime} \neq \mathbf{K}}}\left|C_{\mathbf{K}^{\prime} \boldsymbol{\Phi}^{\prime}, \mathbf{K} \boldsymbol{\Phi}}(t)\right|^{2}\left(E_{\mathbf{K}^{\prime}}-E_{\mathbf{K}}\right)\right) .
\end{gathered}
$$

We approximate the propagator $\hat{U}(t)$ by

$$
\hat{U}(t) \approx \hat{U}_{0}(t)+\frac{1}{\mathrm{i} \hbar} \int_{0}^{t} \hat{U}_{0}(t-s) \hat{V} \hat{U}_{0}(s) \mathrm{d} s .
$$

This is often referred to as the Dyson expansion of the propagator, here approximated to first order. Note that, since $|\mathbf{K} \boldsymbol{\Phi}\rangle$ are the eigenvectors of the unperturbed Hamiltonian, the zeroth order contribution to $C_{\mathbf{K}^{\prime} \boldsymbol{\Phi}^{\prime}, \mathbf{K} \boldsymbol{\Phi}}(t)$ will be zero if $\left|\mathbf{K}^{\prime} \boldsymbol{\Phi}^{\prime}\right\rangle$ is different from the initial eigenstate. Hence, for $\mathbf{K}^{\prime} \neq \mathbf{K}$,

$C_{\mathbf{K}^{\prime} \boldsymbol{\Phi}^{\prime}, \mathbf{K} \boldsymbol{\Phi}}(t) \approx\left\langle\mathbf{K}^{\prime} \boldsymbol{\Phi}^{\prime},\left(\frac{1}{\mathrm{i} \hbar} \int_{0}^{t} \hat{U}_{0}(t-s) \hat{V} \hat{U}_{0}(s) \mathrm{d} s\right) \mathbf{K} \boldsymbol{\Phi}\right\rangle$,

which leads to

$\left|C_{\mathbf{K}^{\prime} \boldsymbol{\Phi}^{\prime}, \mathbf{K} \boldsymbol{\Phi}}(t)\right|^{2}=\frac{t^{2}}{\hbar^{2}}\left|\left\langle\mathbf{K}^{\prime} \boldsymbol{\Phi}^{\prime}, \hat{V} \mathbf{K} \boldsymbol{\Phi}\right\rangle\right|^{2} \operatorname{sinc}^{2}\left(\frac{\left(E_{\mathbf{K}^{\prime} \boldsymbol{\Phi}^{\prime}}-E_{\mathbf{K} \boldsymbol{\Phi}}\right) t}{2 \hbar}\right)$.

We therefore have

$$
\begin{aligned}
& \sum_{\substack{\mathbf{K}^{\prime}, \boldsymbol{\Phi}^{\prime} \\
\mathbf{K}^{\prime} \neq \mathbf{K}}}\left|C_{\mathbf{K}^{\prime} \boldsymbol{\Phi}^{\prime}, \mathbf{K} \boldsymbol{\Phi}}(t)\right|^{2}\left(E_{\mathbf{K}^{\prime}}-E_{\mathbf{K}}\right) \\
& \quad=\frac{t^{2}}{\hbar^{2}} \sum_{\substack{\mathbf{K}^{\prime}, \boldsymbol{\Phi}^{\prime} \\
\mathbf{K}^{\prime} \neq \mathbf{K}}}\left|\left\langle\mathbf{K}^{\prime} \boldsymbol{\Phi}^{\prime}, \hat{V} \mathbf{K} \boldsymbol{\Phi}\right\rangle\right|^{2} \operatorname{sinc}^{2}\left(\frac{\left(E_{\mathbf{K}^{\prime} \boldsymbol{\Phi}^{\prime}}-E_{\mathbf{K} \boldsymbol{\Phi}}\right) t}{2 \hbar}\right)\left(E_{\mathbf{K}^{\prime}}-E_{\mathbf{K}}\right) .
\end{aligned}
$$

Inserting the form of $\hat{V}$ we find that

$$
\begin{aligned}
& \left|\left\langle\mathbf{K}^{\prime} \boldsymbol{\Phi}^{\prime}, \hat{V} \mathbf{K} \boldsymbol{\Phi}\right\rangle\right|^{2} \\
& =\sum_{\mathbf{k}, \mathbf{k}^{\prime}, \lambda}\left|g_{\lambda}\left(\mathbf{k}, \mathbf{k}^{\prime}\right)\right|^{2}\left|\left\langle\mathbf{K}^{\prime}, \hat{c}^{+}\left(\mathbf{k}^{\prime}\right) \hat{c}(\mathbf{k}) \mathbf{K}\right\rangle\right|^{2}\left|\left\langle\boldsymbol{\Phi}^{\prime},\left(\hat{a}_{\lambda}(\mathbf{q})+\hat{a}_{\lambda}^{+}(-\mathbf{q})\right) \boldsymbol{\Phi}\right\rangle\right|^{2}
\end{aligned}
$$




$$
\begin{gathered}
+\sum_{\mathbf{k}, \mathbf{k}^{\prime}, \lambda} \sum_{\substack{1,1^{\prime}, \gamma \\
\left(\mathbf{k}, \mathbf{k}^{\prime}, \lambda\right) \neq\left(\mathbf{1}, \mathbf{1}^{\prime}, \gamma\right)}} g_{\lambda}\left(\mathbf{k}, \mathbf{k}^{\prime}\right) \bar{g}_{\gamma}\left(\mathbf{l}, \mathbf{l}^{\prime}\right)\left\langle\mathbf{K}^{\prime}, \hat{c}^{+}\left(\mathbf{k}^{\prime}\right) \hat{c}(\mathbf{k}) \mathbf{K}\right\rangle \overline{\left\langle\mathbf{K}^{\prime}, \hat{c}^{+}\left(\mathbf{l}^{\prime}\right) \hat{c}(\mathbf{l}) \mathbf{K}\right\rangle} \\
\left\langle\boldsymbol{\Phi}^{\prime},\left(\hat{a}_{\lambda}(\mathbf{q})+\hat{a}_{\lambda}^{+}(-\mathbf{q})\right) \mathbf{\Phi}\right\rangle \overline{\left\langle\boldsymbol{\Phi}^{\prime},\left(\hat{a}_{\gamma}(\mathbf{p})+\hat{a}_{\gamma}^{+}(-\mathbf{p})\right) \boldsymbol{\Phi}\right\rangle} .
\end{gathered}
$$

where $\mathbf{p}=\mathbf{l}^{\prime}-\mathbf{l}+\tilde{\mathbf{k}}$ where $\tilde{\mathbf{k}}$ is a vector of the reciprocal lattice such that lies in the first Brillouin zone. We are thus left with two inner products to evaluate, an electronic one and an ion one. Considering the electronic inner product first, for $\left\langle\mathbf{K}^{\prime}, \hat{c}^{+}\left(\mathbf{k}^{\prime}\right) \hat{c}(\mathbf{k}) \mathbf{K}\right\rangle$ to be non-zero, $\mathbf{K}^{\prime}$ and $\mathbf{K}$ must differ only by an electron-hole pair with momenta $\mathbf{k}$ and $\mathbf{k}^{\prime}$. This means then that the inner product $\left\langle\mathbf{K}^{\prime}, \hat{c}^{+}\left(\mathbf{l}^{\prime}\right) \hat{c}(\mathbf{l}) \mathbf{K}\right\rangle$ will be zero unless $\mathbf{k}^{\prime}=\mathbf{l}^{\prime}$ and $\mathbf{k}=1$. Similarly the ion inner product, $\left\langle\boldsymbol{\Phi}^{\prime},\left(\hat{a}_{\lambda}(\mathbf{q})+\hat{a}_{\lambda}^{+}(-\mathbf{q})\right) \boldsymbol{\Phi}\right\rangle$, will be nonzero only if $\boldsymbol{\Phi}^{\prime}$ and $\boldsymbol{\Phi}$ differ by a single phonon-hole pair. With careful accounting of whether $\left(\mathbf{K}^{\prime} \boldsymbol{\Phi}^{\prime}\right)$ and $(\mathbf{K} \boldsymbol{\Phi})$ differ by the requisite electron-hole and phonon-hole pairs we find that only two terms in the sum (A.2) are non-zero leaving us with

$$
\begin{aligned}
& \sum_{\substack{\mathbf{K}^{\prime}, \boldsymbol{\Phi}^{\prime} \\
\mathbf{K}^{\prime} \neq \mathbf{K}}}\left|C_{\mathbf{K}^{\prime} \boldsymbol{\Phi}^{\prime}, \mathbf{K} \boldsymbol{\Phi}}(t)\right|^{2}\left(E_{\mathbf{K}^{\prime}}-E_{\mathbf{K}}\right) \\
& =\frac{t^{2}}{\hbar^{2}} \sum_{\mathbf{k}, \mathbf{k}^{\prime}, \lambda}\left|g_{\lambda}\left(\mathbf{k}, \mathbf{k}^{\prime}\right)\right|^{2} n_{\mathbf{k}}\left(1-n_{\mathbf{k}^{\prime}}\right)\left(N_{\lambda}(-\mathbf{q})+1\right) \\
& \quad \operatorname{sinc}^{2}\left(\frac{\left(\varepsilon_{\mathbf{k}^{\prime}}-\varepsilon_{\mathbf{k}}+\hbar \omega_{\lambda}(-\mathbf{q})\right) t}{2 \hbar}\right)\left(\varepsilon_{\mathbf{k}^{\prime}}-\varepsilon_{\mathbf{k}}\right) \\
& +\frac{t^{2}}{\hbar^{2}} \sum_{\mathbf{k}, \mathbf{k}^{\prime}, \lambda}\left|g_{\lambda}\left(\mathbf{k}, \mathbf{k}^{\prime}\right)\right|^{2} n_{\mathbf{k}}\left(1-n_{\mathbf{k}^{\prime}}\right) N_{\lambda}(\mathbf{q}) \\
& \operatorname{sinc}^{2}\left(\frac{\left(\varepsilon_{\mathbf{k}^{\prime}}-\varepsilon_{\mathbf{k}}-\hbar \omega_{\lambda}(\mathbf{q})\right) t}{2 \hbar}\right)\left(\varepsilon_{\mathbf{k}^{\prime}}-\varepsilon_{\mathbf{k}}\right) .
\end{aligned}
$$

We now take the large $t$ limit, where the sinc functions narrow to delta functions. Inserting (A.3) in to (A.1) we obtain

$$
\begin{gathered}
\Delta E_{\mathrm{e}}(t)=\frac{2 \pi t}{\hbar} \sum_{\mathbf{k}, \mathbf{k}^{\prime}, \lambda}\left|g_{\lambda}\left(\mathbf{k}, \mathbf{k}^{\prime}\right)\right|^{2} f_{\mathbf{k}}\left(1-f_{\mathbf{k}^{\prime}}\right)\left(\left\langle N_{\lambda}(-\mathbf{q})\right\rangle+1\right) \\
\delta\left(\varepsilon_{\mathbf{k}^{\prime}}-\varepsilon_{\mathbf{k}}+\hbar \omega_{\lambda}(-\mathbf{q})\right)\left(-\hbar \omega_{\lambda}(-\mathbf{q})\right) \\
+\frac{2 \pi t}{\hbar} \sum_{\mathbf{k}, \mathbf{k}^{\prime}, \lambda}\left|g_{\lambda}\left(\mathbf{k}, \mathbf{k}^{\prime}\right)\right|^{2} f_{\mathbf{k}^{\prime}}\left(1-f_{\mathbf{k}}\right)\left\langle N_{\lambda}(\mathbf{q})\right\rangle \\
\delta\left(\varepsilon_{\mathbf{k}}-\varepsilon_{\mathbf{k}^{\prime}}-\hbar \omega_{\lambda}(\mathbf{q})\right)\left(\hbar \omega_{\lambda}(\mathbf{q})\right)
\end{gathered}
$$

having used $\left|g_{\lambda}\left(\mathbf{k}, \mathbf{k}^{\prime}\right)\right|^{2}=\left|g_{\lambda}\left(\mathbf{k}^{\prime}, \mathbf{k}\right)\right|^{2}$ to swap $\mathbf{k}$ and $\mathbf{k}^{\prime}$. Considering just the phonon creation term now, we introduce a sum over all phonon wavevectors and focus on

$$
\begin{aligned}
\sum_{\mathbf{q}} \sum_{\mathbf{k}, \mathbf{k}^{\prime}, \lambda}\left|g_{\lambda}\left(\mathbf{k}, \mathbf{k}^{\prime}\right)\right|^{2} f_{\mathbf{k}}\left(1-f_{\mathbf{k}^{\prime}}\right)\left(\left\langle N_{\lambda}(\mathbf{q})\right\rangle+1\right) \\
\\
\delta\left(\varepsilon_{\mathbf{k}^{\prime}}-\varepsilon_{\mathbf{k}}+\hbar \omega_{\lambda}(\mathbf{q})\right)\left(-\hbar \omega_{\lambda}(\mathbf{q})\right) \delta\left(\mathbf{k}-\mathbf{k}^{\prime}-\tilde{\mathbf{k}}-\mathbf{q}\right) .
\end{aligned}
$$


This is possible since $\tilde{\mathbf{k}}$ is the reciprocal lattice vector required for $\mathbf{q}$ to be in the first Brillouin zone and hence can be considered a function of $\mathbf{k}$ and $\mathbf{k}^{\prime}$. We now write the sum over $\mathbf{k}$ and $\mathbf{k}^{\prime}$ as an integral over different energy surfaces using

$$
\sum_{\mathbf{k}}=\int \mathrm{d} E \int_{S_{E}} \frac{\mathrm{d}^{2} \mathbf{k}}{|\nabla \varepsilon(\mathbf{k})|}
$$

where $S_{E}=\{\mathbf{k} \mid \varepsilon(\mathbf{k})=E\}$ to give

$$
\begin{gathered}
\sum_{\mathbf{q}, \lambda}\left(\left\langle N_{\mathbf{q}, \lambda}\right\rangle+1\right)\left(-\hbar \omega_{\lambda}(\mathbf{q})\right) \int \mathrm{d} E \int \mathrm{d} E^{\prime} f(E)\left(1-f\left(E^{\prime}\right)\right) \delta\left(E^{\prime}-E+\hbar \omega_{\lambda}(\mathbf{q})\right) \\
\int_{S_{E}} \frac{\mathrm{d}^{2} \mathbf{k}}{|\nabla \varepsilon(\mathbf{k})|} \int_{S_{E^{\prime}}} \frac{\mathrm{d}^{2} \mathbf{k}^{\prime}}{\left|\nabla \varepsilon\left(\mathbf{k}^{\prime}\right)\right|}\left|g_{\lambda}\left(\mathbf{k}, \mathbf{k}^{\prime}\right)\right|^{2} \delta\left(\mathbf{k}-\mathbf{k}^{\prime}-\tilde{\mathbf{k}}-\mathbf{q}\right) .
\end{gathered}
$$

Performing the integral over $E^{\prime}$ and shifting the integral over $E$ by $\frac{1}{2} \hbar \omega_{\lambda}(\mathbf{q})$ giving the phonon creation term

$$
\begin{aligned}
& \sum_{\mathbf{q}, \lambda}\left(\left\langle N_{\mathbf{q}, \lambda}\right\rangle+1\right)\left(-\hbar \omega_{\lambda}(\mathbf{q})\right) \\
& \qquad \int \mathrm{d} E f\left(E+\frac{\hbar \omega_{\lambda}(\mathbf{q})}{2}\right)\left(1-f\left(E-\frac{\hbar \omega_{\lambda}(\mathbf{q})}{2}\right)\right) A\left(E, \omega_{\lambda}(\mathbf{q})\right),
\end{aligned}
$$

where $A\left(E, \omega_{\lambda}(\mathbf{q})\right)$ is given by (14). Now performing a similar derivation for the phonon creation term we obtain

$\sum_{\mathbf{q}, \lambda}\left\langle N_{\mathbf{q}, \lambda}\right\rangle \hbar \omega_{\lambda}(\mathbf{q}) \int \mathrm{d} E f\left(E-\frac{\hbar \omega_{\lambda}(\mathbf{q})}{2}\right)\left(1-f\left(E+\frac{\hbar \omega_{\lambda}(\mathbf{q})}{2}\right)\right) A\left(E, \omega_{\lambda}(\mathbf{q})\right)$.

Inserting (A.5) and (A.6) in to (A.4) completes the derivation of (11).

\section{Appendix B. Derivation of Equation (16)}

In this appendix we derive (16) in the long time-limit under the assumption that first-order time-dependent perturbation theory is valid. We consider the Hamiltonian $\hat{H}^{\text {sc }}(t)$ given by $(9)$. We define the corresponding evolution operator

$$
\hat{U}^{\mathrm{sc}}(t)=T \exp \left(-\frac{\mathrm{i}}{\hbar} \int_{0}^{t} \mathrm{~d} s \hat{H}^{\mathrm{sc}}(s)\right)
$$

where $T$ indicates Wick's time-ordering operator. We treat the time-dependent part, denoted $\hat{V}(t)$, as a perturbation to the electronic Hamiltonian, denoted $\hat{H}_{0}$ in this section. Here we are considering only the evolution of the electron system because the ion system is included via $\hat{V}(t)$.

For notational purposes we shall write the perturbation as

$$
\begin{aligned}
& \hat{V}(t)=\sum_{\alpha} \hat{V}_{\alpha}\left(C_{\alpha} \exp \left(-\mathrm{i} \nu_{\alpha} s\right)+D_{\alpha} \exp \left(\mathrm{i} \omega_{\alpha} s\right)\right) \\
& \sum_{\alpha}=\sum_{\mathbf{k}, \mathbf{k}^{\prime}, \lambda}, \quad \hat{V}_{\alpha}=g_{\lambda}\left(\mathbf{k}, \mathbf{k}^{\prime}\right) \hat{c}_{\sigma}^{+}\left(\mathbf{k}^{\prime}\right) \hat{c}_{\sigma}(\mathbf{k}), \\
& C_{\alpha}=\sqrt{N_{\lambda}(\mathbf{q}),} \quad D_{\alpha}=\sqrt{N_{\lambda}(-\mathbf{q})} \\
& \nu_{\alpha}=\omega_{\lambda}(\mathbf{q}), \quad \omega_{\alpha}=\omega_{\lambda}(-\mathbf{q}) .
\end{aligned}
$$


Therefore, using the Dyson expansion for the propagator, for $\mathbf{K}^{\prime} \neq \mathbf{K}$,

$$
\begin{aligned}
c_{\mathbf{K}^{\prime} \mathbf{K}}(t):= & \left\langle\mathbf{K}^{\prime}, \hat{U}(t) \mathbf{K}\right\rangle \approx\left\langle\mathbf{K}^{\prime},\left(\frac{1}{\mathrm{i} \hbar} \int_{0}^{t} \hat{U}_{0}(t-s) \hat{V}(s) \hat{U}_{0}(s)\right) \mathbf{K}\right\rangle \\
= & \frac{1}{\mathrm{i} \hbar} \sum_{\alpha}\left\langle\mathbf{K}^{\prime}, \hat{V}_{\alpha} \mathbf{K}\right\rangle \\
& \quad \int_{0}^{t} \exp \left(-\frac{\mathrm{i} E_{\mathbf{K}^{\prime}}(t-s)}{\hbar}\right)\left(C_{\alpha} \mathrm{e}^{-\mathrm{i} \nu_{\alpha} s}+D_{\alpha} \mathrm{e}^{\mathrm{i} \omega_{\alpha} s}\right) \exp \left(-\frac{\mathrm{i} E_{\mathbf{K}} s}{\hbar}\right) \mathrm{d} s
\end{aligned}
$$

The integral then evaluates to

$$
\begin{aligned}
& C_{\alpha} t \exp \left(-\frac{\mathrm{i} E_{\mathbf{K}^{\prime}} t}{\hbar}\right) \exp \left(\frac{\mathrm{i}\left(E_{\mathbf{K}^{\prime}}-E_{\mathbf{K}}-\hbar \nu_{\alpha}\right) t}{2 \hbar}\right) \operatorname{sinc}\left(\frac{\left(E_{\mathbf{K}^{\prime}}-E_{\mathbf{K}}-\hbar \nu_{\alpha}\right) t}{2 \hbar}\right) \\
& +D_{\alpha} t \exp \left(-\frac{\mathrm{i} E_{\mathbf{K}^{\prime}} t}{\hbar}\right) \exp \left(\frac{\mathrm{i}\left(E_{\mathbf{K}^{\prime}}-E_{\mathbf{K}}+\hbar \omega_{\alpha}\right) t}{2 \hbar}\right) \operatorname{sinc}\left(\frac{\left(E_{\mathbf{K}^{\prime}}-E_{\mathbf{K}}+\hbar \omega_{\alpha}\right) t}{2 \hbar}\right) .
\end{aligned}
$$

We split our evaluation of $\left|c_{\mathbf{K}^{\prime} \mathbf{K}}(t)\right|^{2}$ in to the sum of two parts. The first comes from the product of terms with the same $\alpha$,

$$
\begin{aligned}
& \frac{t^{2}}{\hbar^{2}} \sum_{\alpha}\left|\left\langle\mathbf{K}^{\prime}, \hat{V}_{\alpha} \mathbf{K}\right\rangle\right|^{2} \\
& C_{\alpha}^{2} \operatorname{sinc}^{2}\left(\frac{\left(E_{\mathbf{K}^{\prime}}-E_{\mathbf{K}}-\hbar \nu_{\alpha}\right) t}{2 \hbar}\right)+D_{\alpha}^{2} \operatorname{sinc}^{2}\left(\frac{\left(E_{\mathbf{K}^{\prime}}-E_{\mathbf{K}}+\hbar \omega_{\alpha}\right) t}{2 \hbar}\right) \\
& +C_{\alpha} D_{\alpha} 2 \cos \left(\frac{\left(\hbar \nu_{\alpha}+\hbar \omega_{\alpha}\right) t}{2 \hbar}\right) \\
& \quad \cdot \operatorname{sinc}\left(\frac{\left(E_{\mathbf{K}^{\prime}}-E_{\mathbf{K}}-\hbar \nu_{\alpha}\right) t}{2 \hbar}\right) \operatorname{sinc}\left(\frac{\left(E_{\mathbf{K}^{\prime}}-E_{\mathbf{K}}+\hbar \omega_{\alpha}\right) t}{2 \hbar}\right) .
\end{aligned}
$$

The second, more complex, term comes from the product of terms with different $\alpha$ and will consist of sums of a form similar to

$$
\begin{aligned}
\frac{t^{2}}{\hbar^{2}} \sum_{\alpha} \sum_{\beta \neq \alpha} & \left\langle\mathbf{K}^{\prime}, \hat{V}_{\alpha} \mathbf{K}\right\rangle \overline{\left\langle\mathbf{K}^{\prime}, \hat{V}_{\beta} \mathbf{K}\right\rangle} \\
C_{\alpha} C_{\beta} \exp \left(\frac{\mathrm{i}\left(-\hbar \nu_{\alpha}+\hbar \nu_{\beta}\right) t}{2 \hbar}\right) & \cdot \operatorname{sinc}\left(\frac{\left(E_{\mathbf{K}^{\prime}}-E_{\mathbf{K}}-\hbar \nu_{\alpha}\right) t}{2 \hbar}\right) \operatorname{sinc}\left(\frac{\left(E_{\mathbf{K}^{\prime}}-E_{\mathbf{K}}-\hbar \nu_{\beta}\right) t}{2 \hbar}\right) .
\end{aligned}
$$

We shall that the contribution of terms like (B.2) to $\left|c_{\mathbf{K}^{\prime} \mathbf{K}}\right|^{2}$ goes to zero in the large $t$ limit. From Appendix A we have

$\left\langle\mathbf{K}^{\prime}, \hat{c}^{+}\left(\mathbf{k}^{\prime}\right) \hat{c}(\mathbf{k}) \mathbf{K}\right\rangle \overline{\left\langle\mathbf{K}^{\prime}, \hat{c}^{+}\left(\mathbf{l}^{\prime}\right) \hat{c}(\mathbf{l}) \mathbf{K}\right\rangle}=0$ unless $\mathbf{k}=\mathbf{l}$ and $\mathbf{k}^{\prime}=\mathbf{l}^{\prime}$.

Using this simplification and converting back to the original momenta notation

$$
\begin{aligned}
& \frac{t^{2}}{\hbar^{2}} \sum_{\mathbf{k}, \mathbf{k}^{\prime}} \sum_{\substack{\lambda, \rho \\
\lambda \neq \rho}}\left|g_{\lambda}\left(\mathbf{k}, \mathbf{k}^{\prime}\right)\right|^{2}\left|\left\langle\mathbf{K}^{\prime}, \hat{c}_{\sigma}^{+}\left(\mathbf{k}^{\prime}\right) \hat{c}_{\sigma}(\mathbf{k}) \mathbf{K}\right\rangle\right|^{2} \\
& \left(\sqrt{\left\langle N_{\mathbf{q}, \lambda}\right\rangle} \sqrt{\left\langle N_{\mathbf{q}, \rho}\right\rangle} \exp \left(\frac{\mathrm{i}\left(-\hbar \omega_{\lambda}(\mathbf{q})+\hbar \omega_{\rho}(\mathbf{q})\right) t}{2 \hbar}\right)\right. \\
& \left.\quad \operatorname{sinc}\left(\frac{\left(E_{\mathbf{K}^{\prime}}-E_{\mathbf{K}}-\hbar \omega_{\lambda}(\mathbf{q})\right) t}{2 \hbar}\right) \operatorname{sinc}\left(\frac{\left(E_{\mathbf{K}^{\prime}}-E_{\mathbf{K}}-\hbar \omega_{\rho}(\mathbf{q})\right) t}{2 \hbar}\right)\right)
\end{aligned}
$$


The two sinc functions are centred about energies $\hbar \omega_{\lambda}(\mathbf{q})$ and $\hbar \omega_{\rho}(\mathbf{q})$. If these two energies are different then as time progresses the product of these two sinc functions will tend to zero. If however the two energies are the same, meaning that the frequencies of two different polarisations with the same momentum are equal, then this term will not tend to zero. However this equality of frequencies will only occur for a small number of momenta. Therefore we assume the contribution of (B.2) is zero. A similar argument can be applied to all terms with different $\alpha$.

Therefore only (B.1) contributes to the value of $\left|c_{\mathbf{K}^{\prime} \mathbf{K}}(t)\right|^{2}$. The consideration of whether states $\mathbf{K}^{\prime}$ and $\mathbf{K}$ have the necessary electron-hole pairing to give a non-zero matrix element leads to

$$
\begin{aligned}
& \sum_{\mathbf{K}^{\prime}}\left|c_{\mathbf{K}^{\prime} \mathbf{K}}(t)\right|^{2}\left(E_{\mathbf{K}^{\prime}}-E_{\mathbf{K}}\right) \\
= & \frac{2 \pi t}{\hbar} \sum_{\mathbf{k}, \mathbf{k}^{\prime}, \lambda}\left|g_{\lambda}\left(\mathbf{k}, \mathbf{k}^{\prime}\right)\right|^{2} n_{\mathbf{k}}\left(1-n_{\mathbf{k}^{\prime}}\right)\left\langle N_{-\mathbf{q}, \lambda}\right\rangle \\
& \quad \operatorname{sinc}^{2}\left(\frac{\left(\varepsilon_{\mathbf{k}^{\prime}}-\varepsilon_{\mathbf{k}}+\hbar \omega_{\lambda}(-\mathbf{q})\right) t}{2 \hbar}\right)\left(\varepsilon_{\mathbf{k}^{\prime}}-\varepsilon_{\mathbf{k}}\right) \\
+\frac{2 \pi t}{\hbar} \sum_{\mathbf{k}, \mathbf{k}^{\prime}, \lambda}\left|g_{\lambda}\left(\mathbf{k}, \mathbf{k}^{\prime}\right)\right|^{2} n_{\mathbf{k}}\left(1-n_{\mathbf{k}^{\prime}}\right)\left\langle N_{\mathbf{q}, \lambda}\right\rangle & \operatorname{sinc}^{2}\left(\frac{\left(\varepsilon_{\mathbf{k}^{\prime}}-\varepsilon_{\mathbf{k}}-\hbar \omega_{\lambda}(\mathbf{q})\right) t}{2 \hbar}\right)\left(\varepsilon_{\mathbf{k}^{\prime}}-\varepsilon_{\mathbf{k}}\right) .
\end{aligned}
$$

The derivation now proceeds in a similar fashion to that in Appendix A using

$\Delta E_{\mathrm{e}}^{\mathrm{sc}}(t)=\sum_{\mathbf{K}} \frac{1}{Z_{\mathrm{e}}} \exp \left(-\frac{E_{\mathbf{K}}}{k_{\mathrm{B}} T_{\mathrm{e}}}\right)\left(\sum_{\substack{\mathbf{K}^{\prime} \\ \mathbf{K}^{\prime} \neq \mathbf{K}}}\left|c_{\mathbf{K}^{\prime}, \mathbf{K}}(t)\right|^{2}\left(E_{\mathbf{K}^{\prime}}-E_{\mathbf{K}}\right)\right)$.

\section{Appendix C. Low Temperature Derivation}

We now derive equation (18) and (19) under the assumptions given in section 5.1.

We first evaluate

$$
\begin{aligned}
\mathfrak{A}\left(\omega_{\lambda}(\mathbf{q})\right)-\mathfrak{E}\left(\omega_{\lambda}(\mathbf{q})\right) & \\
:= & \int_{B} \mathrm{~d} E f\left(E-\frac{\hbar \omega_{\lambda}(\mathbf{q})}{2}\right)\left(1-f\left(E+\frac{\hbar \omega_{\lambda}(\mathbf{q})}{2}\right)\right) A\left(E, \omega_{\lambda}(\mathbf{q})\right) \\
& -\int_{B} \mathrm{~d} E f\left(E+\frac{\hbar \omega_{\lambda}(\mathbf{q})}{2}\right)\left(1-f\left(E-\frac{\hbar \omega_{\lambda}(\mathbf{q})}{2}\right)\right) A\left(E, \omega_{\lambda}(\mathbf{q})\right),
\end{aligned}
$$

where $B$ denotes the electronic bandwidth. By the first assumption of section 5.1, $A\left(E, \omega_{\lambda}(\mathbf{q})\right)$ is constant over the energy range where the integrand is non-zero. This allows us to take this system dependent term outside of the integral. By the second assumption the integrand is non-zero only for a small region of the bandwidth $B$. We can therefore extend the integral over the bandwidth to one from $-\infty$ to $+\infty$. We are thus left with the following integral to evaluate,

$$
\int_{-\infty}^{+\infty} \mathrm{d} E\left(f\left(E-\frac{\hbar \omega}{2}\right)\left(1-f\left(E+\frac{\hbar \omega}{2}\right)\right)-f\left(E+\frac{\hbar \omega}{2}\right)\left(1-f\left(E-\frac{\hbar \omega}{2}\right)\right)\right)
$$


The Ehrenfest approximation for electrons and phonons

$$
\begin{aligned}
=\frac{1}{2}\left(\exp \left(\frac{\hbar \omega}{2 k_{\mathrm{B}} T_{\mathrm{e}}}\right)-\exp \left(-\frac{\hbar \omega}{2 k_{\mathrm{B}} T_{\mathrm{e}}}\right)\right) \\
\quad \int_{-\infty}^{+\infty} \mathrm{d} E\left(\cosh \left(\frac{E}{k_{\mathrm{B}} T_{\mathrm{e}}}\right)+\cosh \left(\frac{\hbar \omega}{2 k_{\mathrm{B}} T_{\mathrm{e}}}\right)\right)^{-1},
\end{aligned}
$$

where we have used $\omega=\omega_{\lambda}(\mathbf{q})$ to simplify notation and inserted the definition of the Fermi function. We can rewrite the integral as

$$
\begin{aligned}
\int_{-\infty}^{+\infty} \mathrm{d} E \exp \left(\frac{E}{k_{\mathrm{B}} T_{\mathrm{e}}}\right) \\
\quad\left(\exp \left(\frac{2 E}{k_{\mathrm{B}} T_{\mathrm{e}}}\right)+\exp \left(\frac{E}{k_{\mathrm{B}} T_{\mathrm{e}}}\right)\left(\exp \left(\frac{\hbar \omega}{2 k_{\mathrm{B}} T_{\mathrm{e}}}\right)+\exp \left(-\frac{\hbar \omega}{2 k_{\mathrm{B}} T_{\mathrm{e}}}\right)\right)+1\right)
\end{aligned}
$$

and then by making the substitution the substitution $u=\exp \left(\frac{E}{k_{\mathrm{B}} T_{\mathrm{e}}}\right)$ we have

$$
\begin{aligned}
& k_{\mathrm{B}} T_{\mathrm{e}} \int_{0}^{\infty} \mathrm{d} u\left(u^{2}+u\left(\exp \left(\frac{\hbar \omega}{2 k_{\mathrm{B}} T_{\mathrm{e}}}\right)+\exp \left(-\frac{\hbar \omega}{2 k_{\mathrm{B}} T_{\mathrm{e}}}\right)\right)+1\right)^{-1} \\
= & k_{\mathrm{B}} T_{\mathrm{e}}\left(\exp \left(\frac{\hbar \omega}{2 k_{\mathrm{B}} T_{\mathrm{e}}}\right)+\exp \left(-\frac{\hbar \omega}{2 k_{\mathrm{B}} T_{\mathrm{e}}}\right)\right)^{-1}\left[\ln \left(\frac{u+\exp \left(-\frac{\hbar \omega}{2 k_{\mathrm{B}} T_{\mathrm{e}}}\right)}{u+\exp \left(\frac{\hbar \omega}{2 k_{\mathrm{B}} T_{\mathrm{e}}}\right)}\right)\right]_{0}^{\infty} \\
= & \hbar \omega\left(\exp \left(\frac{\hbar \omega}{2 k_{\mathrm{B}} T_{\mathrm{e}}}\right)+\exp \left(-\frac{\hbar \omega}{2 k_{\mathrm{B}} T_{\mathrm{e}}}\right)\right)^{-1} .
\end{aligned}
$$

From (C.2) we insert the evaluation of the integral given by (C.3) to obtain

$$
\begin{array}{rl}
\int_{-\infty}^{+\infty} \mathrm{d} & E\left(f\left(E-\frac{\hbar \omega}{2}\right)\left(1-f\left(E+\frac{\hbar \omega}{2}\right)\right)\right. \\
\left.-f\left(E+\frac{\hbar \omega}{2}\right)\left(1-f\left(E-\frac{\hbar \omega}{2}\right)\right)\right)=\hbar \omega .
\end{array}
$$

Inserting in to (C.1) we obtain (18).

To derive (19) we need to evaluate

$\mathfrak{E}\left(\omega_{\lambda}(\mathbf{q})\right)=\int_{B} \mathrm{~d} E f\left(E+\frac{\hbar \omega_{\lambda}(\mathbf{q})}{2}\right)\left(1-f\left(E-\frac{\hbar \omega_{\lambda}(\mathbf{q})}{2}\right)\right) A\left(E, \omega_{\lambda}(\mathbf{q})\right)$.

Making the same approximations as for $\mathfrak{A}\left(\omega_{\lambda}(\mathbf{q})\right)-\mathfrak{E}\left(\omega_{\lambda}(\mathbf{q})\right)$ we obtain

$\mathfrak{E}\left(\omega_{\lambda}(\mathbf{q})\right)=A\left(E_{0}, \omega_{\lambda}(\mathbf{q})\right) \int_{-\infty}^{+\infty} \mathrm{d} E f\left(E+\frac{\hbar \omega_{\lambda}(\mathbf{q})}{2}\right)\left(1-f\left(E-\frac{\hbar \omega_{\lambda}(\mathbf{q})}{2}\right)\right)$.

Using the definition of the Fermi function we rewrite the integral as

$\frac{1}{2} \exp \left(-\frac{\hbar \omega}{2 k_{\mathrm{B}} T_{\mathrm{e}}}\right) \int_{-\infty}^{+\infty} \mathrm{d} E\left(\cosh \left(\frac{E}{k_{\mathrm{B}} T_{\mathrm{e}}}\right)+\cosh \left(\frac{\hbar \omega}{2 k_{\mathrm{B}} T_{\mathrm{e}}}\right)\right)^{-1}$,

again relpacing $\omega_{\lambda}(\mathbf{q})$ by $\omega$ to simplify notation. Eq (C.3) gives the evaluation of this integral enabling us to obtain

$$
\mathfrak{E}\left(\omega_{\lambda}(\mathbf{q})\right)=A\left(E_{0}, \omega_{\lambda}(\mathbf{q})\right) \hbar \omega_{\lambda}(\mathbf{q}) \frac{1}{\exp \left(\frac{\hbar \omega_{\lambda}(\mathbf{q})}{k_{\mathrm{B}} T_{\mathrm{e}}}\right)-1} .
$$

This completes the derivation of (19) 


\section{Appendix D. High Temperature Derivation}

We first derive (23). We shall use the following high temperature result

$$
\begin{aligned}
& f\left(E-\frac{\hbar \omega}{2}\right)-f\left(E+\frac{\hbar \omega}{2}\right) \\
= & \frac{1}{2}\left(\exp \left(\frac{\hbar \omega}{2 k_{\mathrm{B}} T_{\mathrm{e}}}\right)-\exp \left(-\frac{\hbar \omega}{2 k_{\mathrm{B}} T_{\mathrm{e}}}\right)\right)\left(\cosh \left(\frac{E}{k_{\mathrm{B}} T_{\mathrm{e}}}\right)+\cosh \left(\frac{\hbar \omega}{2 k_{\mathrm{B}} T_{\mathrm{e}}}\right)\right)^{-1} \\
= & \frac{1}{4} \frac{\hbar \omega}{k_{\mathrm{B}} T_{\mathrm{e}}}
\end{aligned}
$$

where we have used

$$
\exp (x)=1+x \text { for small } x \text { and } \cosh (x)=1 \text { for small } x
$$

and neglected quadratic terms. Now inserting (D.1) in to the definition of $\mathfrak{A}\left(\omega_{\lambda}(\mathbf{q})\right)-$ $\mathfrak{E}\left(\omega_{\lambda}(\mathbf{q})\right)$ given by $($ C.1 $)$ we have

$$
\left(\mathfrak { A } \left(\omega_{\lambda}(\mathbf{q})-\mathfrak{E}\left(\omega_{\lambda}(\mathbf{q})\right)=\frac{1}{4} \frac{\hbar \omega_{\lambda}(\mathbf{q})}{k_{\mathrm{B}} T_{\mathrm{e}}} \int_{B} \mathrm{~d} E A\left(E, \omega_{\lambda}(\mathbf{q})\right),\right.\right.
$$

where no energy dependence in the case of high temperature means we are able to move the Fermi-Dirac function contribution out of the energy integral.

We now derive (24). In this case the Fermi-Dirac functions simplify, through the use of (D.2), to give

$$
\begin{aligned}
& f\left(E+\frac{\hbar \omega}{2}\right)\left(1-f\left(E-\frac{\hbar \omega}{2}\right)\right) \\
= & \frac{1}{2} \exp \left(-\frac{\hbar \omega}{2 k_{\mathrm{B}} T_{\mathrm{e}}}\right)\left(\cosh \left(\frac{E}{k_{\mathrm{B}} T_{\mathrm{e}}}\right)+\cosh \left(\frac{\hbar \omega}{2 k_{\mathrm{B}} T_{\mathrm{e}}}\right)\right)^{-1} \\
= & \frac{1}{4}\left(1-\frac{\hbar \omega}{2 k_{\mathrm{B}} T_{\mathrm{e}}}\right) .
\end{aligned}
$$

Inserting this definition in to the definition of $\mathfrak{E}\left(\omega_{\lambda}(\mathbf{q})\right)$ from (Appendix $C$ ) we have

$$
\mathfrak{E}\left(\omega_{\lambda}(\mathbf{q})\right)=\frac{1}{4}\left(1-\frac{\hbar \omega_{\lambda}(\mathbf{q})}{2 k_{\mathrm{B}} T_{\mathrm{e}}}\right) \int \mathrm{d} E A\left(E, \omega_{\lambda}(\mathbf{q})\right),
$$

where again the lack of explicit energy dependence in the Fermi-Dirac term allows the temperature dependence to be remove from the energy integral.

\section{References}

[1] John B. Delos, Walter R. Thorson, and Stephen K. Knudson. Semiclassical theory of inelastic collisions. I. Classical picture and semiclassical formulation. Phys. Rev. A, 6(2):709-720, Aug 1972.

[2] John C. Tully and Richard K. Preston. Trajectory surface hopping approach to nonadiabatic molecular collisions: The reaction of $\mathrm{H}^{+}$with $\mathrm{D}_{2}$. The Journal of Chemical Physics, $55(2): 562-572,1971$

[3] Andrew P Horsfield, D R Bowler, A J Fisher, Tchavdar N Todorov, and Cristián G Sánchez. Correlated electron-ion dynamics: The excitation of atomic motion by energetic electrons. Journal of Physics: Condensed Matter, 17(30):4793-4812, 2005.

[4] E.J. Heller. Guided gaussian wave packets. Accounts of Chemical Research, 39(2):127-134, 2006.

[5] D M Duffy and A M Rutherford. Including the effects of electronic stopping and electronion interactions in radiation damage simulations. Journal of Physics: Condensed Matter, 19(1):016207 (11pp), 2007. 
[6] Richard P. Feynman. Statistical Mechanics. Advanced Book Classics. Westview Press, 1972.

[7] G. Rickayzen. Green's Functions and Condensed Matter, volume 5 of Techniques of Physics. Academic Press, 1980.

[8] L. J. Sham and J. M. Ziman. The electron-phonon interation. Solid State Physics, 15:221-298, 1963.

[9] John R. Klauder and E. C. G. Sudarshan. Fundamentals of Quantum Optics. Benjamin, New York, 1968

[10] John R. Klauder and Bo-Sture Skagerstam. Coherent States: Applications in Physics and Mathematical Physics. World Scientific Pub Co Inc, 1985.

[11] Jon H. Shirley. Solution of the schrödinger equation with a hamiltonian periodic in time. Phys. Rev., 138(4B):B979-B987, May 1965.

[12] G. A. Raggio and H. S. Zivi. On the semiclassical description of n-level systems interacting with radiation fields. Journal of Mathematical Physics, 26(10):2529-2539, 1985.

[13] S Guérin, F Monti, J-M Dupont, and H R Jauslin. On the relation between cavity-dressed states, floquet states, rwa and semiclassical models. Journal of Physics A: Mathematical and General, 30(20):7193-7215, 1997.

[14] D R Mason, J le Page, C P Race, W M C Foulkes, M W Finnis, and A P Sutton. Electronic damping of atomic dynamics in irradiation damage of metals. Journal of Physics: Condensed Matter. 